\title{
Comportamiento energético de mosaicos urbanos representativos (La Plata-Buenos Aires- Argentina): influencia de las variaciones térmicas intraurbanas
}

\author{
Energetic behavior of representative urban mosaics (La \\ Plata-Buenos Aires-Argentina): the influence of intra- \\ urban thermal variations
}

\section{Graciela Melisa Viegas \\ Pedro J oaquín Chevez \\ Gustavo Alberto San J uan \\ Carlos Alberto Discoli}

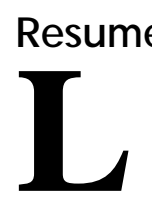

a carga térmica para climatización de edificios (Q) estima la demanda de energía en función de un coeficiente global de pérdidas $(G)$ y de los grados día (GD) de refrigeración o calefacción. En general los GD se calculan con las temperaturas medias publicadas por organismos oficiales. Sin embargo, se ha demostrado que la ciudad afecta al clima urbano generando variaciones térmicas debido a sus condiciones morfológicas y materiales. Este trabajo analiza las condiciones térmicas externas en distintas zonas representativas (mosaicos urbanos [MU]) de la ciudad de La Plata para determinar cómo modifican las condiciones de demanda de energía teórica para climatización. La metodología involucra el registro de la temperatura exterior de diez sectores urbanos; el cálculo de los GD de calefacción $\left(\mathrm{GD}_{18}\right)$ y de refrigeración $\left(\mathrm{GD}_{23}\right)$; la comparación con los GD oficiales; el agrupamiento por zonas urbanas mediante técnicas de clustering de k-medias; y el cálculo de la demanda de energía teórica. Los resultados muestran que la demanda de energía calculada con las mediciones experimentales está sobrestimada en invierno y subestimada en verano comparado con el cálculo realizado con los datos oficiales. Asimismo, se encontraron distribuciones térmicas territoriales diferenciadas entre invierno y verano.

Palablas-clave: Condiciones térmicas urbanas. Grados día calefacción y de refrigeración. Demanda energética teórica. Mosaicos urbanos.

Graciela Melisa Viegas IUniversidad Nacional de La Plata La Plata - Buenos Aires - Argentina

Pedro J oaquín Chevez Universidad Nacional de La Plata La Plata - Buenos Aires - Argentina

Gustavo Alberto San J uan Universidad Nacional de La Plata La Plata - Buenos Aires - Argentina

Carlos Alberto Discoli Universidad Nacional de La Plata La Plata - Buenos Aires - Argentina

Recebido em 23/11/17 Aceito em 28/03/18

\section{Abstract}

Simplified HVAC models for buildings estimate energy demand based on a global loss coefficient $(G)$ and the heating and cooling degree days $(D D)$. The DD are calculated, from citie's average temperatures, published by official entities. However different researches have recorded that the city affects the urban climate with thermal variations due to its morphological and material conditions. This paper analyzes the external thermal situations in different representative areas of La Plata city to determine the degree to which the theoretical energy is demanded for thermal conditioning at the intra-urban level are modified. To address the research, the external temperatures of ten urban sectors were measured. Then, the $D D$ were calculated and compared with the official records, different zones were grouped by the k-means technique and, finally, theoretical energy demand was calculated. Results show that energy demand calculated from experimental measurements is overestimated in winter and underestimated in summer regarding the calculation from official measurements. Also, different territorial thermal distributions were found between winter and summer.

Keywords: Urban thermal conditions. Heating and cooling degree days. Energy performance. Urban mosaics.

Construído, Porto Alegre, v. 18, n. 3, p. 175-194, jul./set. 2018.

ISSN 1678-8621 Associação Nacional de Tecnologia do Ambiente Construído.

http:/ / dx. doi. org/ 10.1590/ s1678-86212018000300275 


\section{Introducción}

A nivel mundial, el consumo de energía del sector residencial tiene una participación significativa sobre el consumo de energía total, cuya incidencia es cercana al 23\% (INTERNATIONAL..., 2015). En el caso de Argentina, el sector residencial demanda aproximadamente el $27 \%$ de la energía total utilizada en el país, lo que lleva a situaciones de competencia entre sectores y, en particular, entre el sector residencial y el industrial, que la requiere con fines productivos, demandando un 22,5\% de la energía total (MINISTERIO..., 2017).

Con el objetivo de reducir la demanda de energía por parte del sector residencial, se han estudiado y desarrollado en el mundo diversas estrategias basadas en la aplicación de medidas de eficiencia energética e incorporación de energías renovables. En este sentido, se entiende que necesariamente se deben aplicar estrategias que respondan a criterios de intervención masiva sobre la ciudad y no a soluciones parciales sobre edificios aislados (DÍSCOLI; MARTINI, 2012). Por lo tanto, resulta necesario desarrollar modelos simplificados que permitan estimar situaciones "de base" de demanda de energía y, en función de ello, estimar posibles ahorros a partir de las características edilicias del sector residencial. En general, los modelos de estimación se basan en el cálculo de demanda y ahorro en edificios individuales. Pero muy pocos abordan la escala urbana, la cual se presenta como un área de vacancia para las investigaciones.

Con relación a dicha temática, se han desarrollado modelos a nivel internacional que tienen por objetivo analizar la performance energética de sectores urbanos y de ciudades. Estos modelos permiten evaluar la aplicación de estrategias de intervención para mejorar las condiciones actuales, reducir el consumo de energía y el impacto ambiental. En Rodríguez Álvarez (2016) se desarrolla una compilación de los últimos modelos energéticos urbanos. En lo que refiere a los modelos térmicos estacionarios, podemos encontrar el Energy and Environmental Prediction model (EEP), el LT model, el Climate Lite, el Ursos, el GDR energy model. y el Urban Energy Building Index (UEBI). Este último se destaca debido a que considera la influencia de la morfología ediliciaurbana en la performance energética de la ciudad, a partir de la construcción de un patrón regular adoptando los promedios geométricos de la morfología existente. Asimismo, considera

${ }^{1}$ Energía que pierde un local calefaccionado por unidad de volumen, unidad de tiempo y unidad de diferencia de temperatura, en régimen estacionario, y que deberá suplir e sistema de calefacción para mantener constante la temperatura interna del local -Norma IRAM 11604/ 01-(INSTITUTO..., 2001). parámetros por default (tipo de materiales y ocupación edilicia) y no considera situaciones térmicas diferenciadas de la ciudad. También existen modelos basados en simulaciones térmicas dinámicas, como es el caso del SunTool y del UMI (Urban Modeling Interface). Se puede concluir que el éxito de los modelos estudiados radica en su simplicidad y en la capacidad de ser utilizados en distintas localizaciones urbanas; sin embargo, no consideran la heterogeneidad térmica de cada sector de la ciudad en su cálculo de demanda energética. En consecuencia, los errores de déficit o de exceso en el cálculo de la energía de demanda, pueden ser significativos.

Para el caso de estudio de este trabajo, se adoptan cálculos validados de estimación de la-demanda de energía para edificios individuales, en función de la aplicación de normas nacionales de carácter no obligatorio (INSTITUTO..., 1996a, 1996b), en las cuales se desarrollan modelos estacionarios basados en la demanda de energía (Q) para climatización a partir del coeficiente volumétrico de pérdidas de calor $\left(\mathrm{G}^{1}\right)$. Dichas normas estiman el Q utilizando los grados día $\left(\mathrm{GD}^{2}\right)$ de invierno y verano de la ciudad seleccionada, que se calculan a partir de la recolección y tratamiento de los datos meteorológicos que publica el Servicio Meteorológico Nacional (organismo oficial, http://www.smn.gov.ar/). Con base en esta normativa y buscando avanzar sobre metodologías que involucren sectores urbanos, se desarrolló un modelo que está basado en el análisis de la potencialidad energética de distintos sectores de la ciudad, los cuales se denominan MU. En el modelo basado en MU, se aplican estimaciones de cálculo en estado estacionario propuestas por las normas IRAM (VIEGAS, 2011; VIEGAS; SAN JUAN, 2012), a su vez, se profundiza sobre los aspectos morfológicos del entorno urbano y es posible comparar sectores de diferente compacidad y accesibilidad solar.

La mayoría de los modelos descriptos se basan en el uso de parámetros unificados a nivel urbano para su aplicación. Pero si consideramos que las ciudades se disponen sobre el territorio de forma extensiva e intensiva a partir de su densidad urbana, en diversas condiciones geomorfológicas, paisajísticas y edilicio-morfológicas, se entiende la importancia de considerar que los parámetros climáticos requieren ser desagregados y analizados.
${ }^{2}$ Suma de las diferencias de temperaturas - entre una temperatura base y la media diaria- para los días en los que la media diaria es menor o mayor (según período invernal o estival) a la temperatura base en un período establecido -Norma IRAM 11549/ 02-(INSTITUTO..., 2002) 
En este sentido, numerosas investigaciones han observado y estudiado las diferencias térmicas que se presentan en distintas áreas de las ciudades, con sus consecuentes efectos sobre la demanda energética y la condición de confort ambiental (WUDAPT, 2017; CAI et al., 2017; VERDONCK et al., 2011; BROUSSE et al., 2016; SOSA; CORREA; CANTÓN, 2016a). A partir de esta observación, se realizan aproximaciones teóricas o empíricas sobre las condiciones térmicas de distintas zonas urbanas.

En este sentido, se destaca la iniciativa del World Urban Database (WUDAPT, 2017), que reúne información de numerosas ciudades, sobre las cuales es posible consultar o procesar información para obtener lo que se denominan "zonas climáticas locales” (en inglés, local climate zone [LCZ]). Las LCZ poseen determinadas características climáticas que se obtienen a partir de la forma y la función de los distintos sectores urbanos. En la revisión de la literatura, es posible detectar numerosos ejemplos que aplican el mencionado modelo, el cual, en ciertos casos, es contrastado con otros tipos de aproximaciones, ya sean empíricas o teóricas (CAI et al., 2017; VERDONCK et al., 2011; BROUSSE et al., 2016). Asimismo, se encuentran otros trabajos que evalúan el comportamiento térmico intraurbano, como el de De Ridder et al. (2015), en el que se desarrolla el modelo UrbClim y se lo evalúa en ciudades de Francia, Bélgica y España. Por su parte, Lee y Oh (2017) clasifican zonas climáticas dentro de la ciudad a partir de un acercamiento estadístico, utilizando información de 246 estaciones meteorológicas ubicadas en la ciudad de Seúl, Corea del Sur (37³3'N, 12658'E, $50 \mathrm{msnm}$ ). Por su parte, se puede observar que los trabajos de Palme et al. (2017a, 2017b) y Palme, Carrasco y Galvez (2016) realizan simulaciones climáticas utilizando el software Urban Weather Generator (BUENO et al., 2013), generando archivos de clima aplicables a programas de simulación dinámica de edificios para calcular la demanda de energía teórica para refrescamiento. Sus aplicaciones en ciudades costeras latinoamericanas han arrojado valores de subestimación de entre un 15 y un 200\% de la demanda de energía para refrigeración de edificios.

Por otra parte, otros estudios se focalizan sobre los datos empíricos registrados en los diferentes sectores urbanos. Es el caso de Ochoa y Serra (1998), quienes analizan dos sectores urbanos de Barcelona para evaluar la influencia de la vegetación en el comportamiento microclimático. Asimismo, Gallinelli, Caponovo y Guillot (2017) remarcan la necesidad de contar con mediciones urbanas específicas, en contraposición a la información proveniente de estaciones meteorológicas. El trabajo expone el desarrollo de un sensor de medición para hacer "caminatas climáticas urbanas” y, así, evaluar la incidencia de diversas variables sobre el clima urbano en Génova-

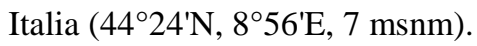

Con relación a esta metodología, el equipo de investigación del IIPAC-FAU-UNLP ${ }^{3}$ desarrolló un Atlas Urbano Ambiental (AUA) para la ciudad de La Plata-Argentina (345' ${ }^{\circ}$, 57 $7^{\circ} 57^{\prime} \mathrm{O}, 17$ msnm). El AUA permitió analizar el fenómeno de isla de calor urbana (ICU), que se produce por cambios en la temperatura de la atmósfera en la ciudad debido a las características de la urbanización y que define sus condiciones a partir de mediciones de la temperatura ambiente y la del pavimento en corredores urbanos característicos que conectan la ciudad con la periferia. Si bien se registraron diferencias térmicas en los sectores urbanos, no se observaron niveles térmicos que excedieran por tiempo prolongado a los de referencia. Se concluyó que la ciudad no presentó perfiles térmicos relacionados con el fenómeno de ICU (DÍSCOLI, 2009), aunque sí se verificó la existencia de diferencias térmicas significativas.

Continuando con las mediciones empíricas, en la ciudad de Mendoza-Argentina (3253'S, 6850'O, $772 \mathrm{msnm}$ ), el trabajo de Correa, De Rosa y Lesino (2008) afirma que el efecto de la ICU modifica los grados día de calefacción y refrigeración en distintos puntos de la ciudad detectando sobrestimaciones del 50\% en GD de calefacción y subestimaciones del 9\% en GD de refrigeración. Posteriormente — sobre la misma ciudad - en los trabajos de Sosa, Correa y Cantón (2016a, 2016b), se tomaron mediciones de temperatura de verano en nueve corredores urbanos y se relevaron sus características morfológicas, vegetales y espaciales para obtener un modelo predictivo de temperatura máxima y también identificar formas urbanas que contribuyan a reducir el efecto de la ICU en dicha ciudad.

Finalmente, se encuentran trabajos que avanzan sobre el análisis de las condiciones térmicas existentes en una ciudad y se evalúa la aplicación de medidas de mejoramiento climático urbano. Tal es el caso de Zölch et al. (2016), en el que se presenta un análisis del impacto de diferentes medidas de mitigación de "infraestructura urbana verde”, como árboles, techos verdes o fachadas verdes, sobre el confort térmico de los peatones. Sin embargo, los

Instituto de Investigaciones y Políticas del Ambiente Construido de la Facultad de Arquitectura y Urbanismo, Universidad Nacional de La Plata, Argentina. 
efectos son simulados a partir de información climática proveniente de un punto de medición diferente al sector urbano. Otro ejemplo es el de Park et al. (2017), trabajo que simula los cambios ocurridos en el flujo de aire y temperatura luego de aplicar programas de tecnologías de regeneración de infraestructura urbana en una ciudad de Corea del Sur. Por su parte, en Fabbri et al. (2017) y en Gaspari y Fabbri (2017), se simulan las condiciones microclimáticas de un sector urbano de Bolonia con el software ENVI-MET, a los efectos de diseñar los espacios exteriores con el mayor grado de confort posible. Sin embargo, no se modela con temperaturas específicas del sector urbano de estudio. Lo mismo sucede en el trabajo de Li, Wang y Wong (2016), que analiza la forma urbana y las condiciones microclimáticas en días de verano, pero realiza la simulación con una única información climática.

Los antecedentes muestran que tanto los estudios basados en simulación numérica como en mediciones experimentales del comportamiento térmico de corredores o sectores urbanos, se orientan preferentemente al diagnóstico para el mejoramiento del confort ambiental de estos. Son menos frecuentes los estudios tendientes a la reducción de la carga térmica de la edificación a partir de la utilización de modelos simplificados de cálculo de demanda de energía. En consecuencia, se observa una exigua relación entre los modelos energéticos simplificados y los análisis de temperaturas urbanas. En este sentido, integrar ambos avances en una nueva investigación representa un abordaje novedoso y de amplia relevancia. Por otro lado, no se observaron estudios que incluyan un análisis integral de las condiciones térmicas de verano e invierno en diferentes regiones urbanas, mientras que son escasos aquellos que buscan obtener una territorialización que permita relacionarla con mapeos energéticos.
En este contexto, el objetivo del presente trabajo es el de integrar el desarrollo de modelos simplificados orientados a calcular la demanda de energía para climatización (o carga térmica) de edificaciones en sectores urbanos característicos, basado en el concepto de MU, utilizando las condiciones térmicas externas a los efectos de minimizar los errores de cálculo. El trabajo plantea determinar la diferencia entre la demanda de energía teórica para la climatización edilicia calculada a partir de:

(a) mediciones oficiales provenientes de estaciones meteorológicas; $y$

(b) mediciones experimentales en distintos sectores urbanos.

Esto permite la detección de sectores de mayor demanda energética específica $\mathrm{y}$, con ello, el direccionamiento de medidas de mejoramiento particulares para cada sector definido.

\section{Metodología para la determinación de la demanda energética de sectores urbanos en función del clima}

Se utiliza la ciudad de La Plata como caso de estudio. Se trata de una ciudad intermedia, que contaba con una población de 698.164 habitantes en el año 2017 (INSTITUTO..., 2017). La Plata se ubica en el Noreste de la provincia de Buenos Aires y a 60 km de la Ciudad Autónoma de Buenos Aires (CABA).

La ciudad ocupa una superficie de $821 \mathrm{~km}^{2}$, se caracteriza por la situación geomorfológica de llanura pampeana (HURTADO, 2006) y se localiza en una región climática definida como templada cálida húmeda (INSTITUTO..., 2012), cercana al Río de la Plata. El clima es benigno, aunque sus inviernos y veranos requieren de climatización para alcanzar temperaturas de confort (ver Figura 2).

Tabla 1 - Condiciones climáticas de la ciudad de La Plata

\begin{tabular}{|c|c|c|c|c|c|c|c|c|c|}
\hline \multirow{2}{*}{$\begin{array}{l}\text { Ciudad de } \\
\text { La Plata }\end{array}$} & Lat. & Long. & Altitud & $\begin{array}{c}\mathrm{T} \\
\text { media }\end{array}$ & $\begin{array}{c}\mathbf{T} \\
\text { máx }\end{array}$ & $\begin{array}{c}\mathrm{T} \\
\mathrm{min}\end{array}$ & HR & Lluvia & $\begin{array}{c}\text { Grado } \\
\text { día }\end{array}$ \\
\hline & $\circ$ & $\circ$ & msnm & ${ }^{\circ} \mathrm{C}$ & ${ }^{\circ} \mathbf{C}$ & ${ }^{\circ} \mathrm{C}$ & $\%$ & $\mathbf{m m}$ & GD \\
\hline Invierno & \multirow{2}{*}{$\begin{array}{c}- \\
34,97\end{array}$} & \multirow{2}{*}{$-57,9$} & \multirow{2}{*}{23} & 11,0 & 15,7 & 6,5 & 83 & 264 & $\begin{array}{l}1210 \\
\text { (base } \\
18^{\circ} \mathrm{C} \text { ) }\end{array}$ \\
\hline Verano & & & & 21,8 & 27,4 & 16,4 & 76 & 446 & $\begin{array}{c}606 \\
\text { (base } \\
23^{\circ} \mathrm{C} \text { ) }\end{array}$ \\
\hline
\end{tabular}

Fuente: Norma IRAM 11603 (INSTITUTO..., 2012). 
Para el análisis se seleccionaron diez MU, que son sectores urbanos que representan las características de un área mayor. Dichas áreas poseen 'consolidaciones diferenciadas'. Para definir el área residencial urbana consolidada, se adoptó la metodología presentada en Brea et al. (2014), en la que, para evaluar su graduación, se analiza la intensidad de ocupación del suelo a partir de la densidad poblacional y edilicia y de la cantidad de servicios de infraestructura urbana que se disponen en el área estudiada. Finalmente, se adopta una clasificación de las siguientes tres categorías: consolidación baja, consolidación media y consolidación alta.

La Figura 1 muestra el mapa con la localización de los MU. Éstos fueron distribuidos en las distintas zonas de la ciudad, abarcando tanto el área central, denominada Casco Fundacional (CF), de la ciudad de La Plata como el de su periferia. Se decidió seleccionar áreas de consolidación alta (zona del $\mathrm{CF}$ ), de consolidación media (periferia cercana al CF) y de consolidación baja (áreas periféricas y de borde urbano-rural). En la Figura 1 se observa el CF, la extensión de la periferia, y los puntos de medición (MU).

Por su parte, la Figura 3 presenta imágenes y algunas características de los MU.
La metodología adoptada en este trabajo se desarrolla en las siguientes cuatro etapas: mediciones de temperatura exterior de los MU, método de cálculo de grados día mensuales $\left(\mathrm{GD}_{\mathrm{mes}}\right)$ con datos oficiales y experimentales, identificación de zonas de similar comportamiento térmico y estimación de las demandas de energía teórica a partir de GD oficiales y experimentales.

\section{Mediciones de temperatura exterior de los mosaicos urbanos}

Se realizó una auditoria térmica-energética de los diez MU seleccionados. Para ello, en cada uno se seleccionó una vivienda y se instaló un micro adquisidor electrónico de registro de datos (tipo Hobo) en el entorno externo, resguardado de la radiación solar y a $2 \mathrm{~m}$ de altura sobre nivel de suelo. Además, se constataron los registros climáticos oficiales de la estación meteorológica Observatorio (OALP) y los registros históricos de la estación meteorológica Aeropuerto (ALP), para luego ser contrastados con las mediciones experimentales. Para las mediciones se registraron las condiciones de invierno (desde el 15 de junio hasta el 15 de julio) y de verano (del $1^{\circ}$ al 31 de enero) del año 2012 en intervalos de medición de 15 minutos. Los diez puntos de medición y las dos estaciones meteorológicas se observan en la Figura 1, presentada anteriormente.

\section{Figura 1 - Partido de La Plata: localización de los puntos de medición (MU) y las estaciones meteorológicas Observatorio y Aeropuerto La Plata}




Figura 2 - Imágenes y características de los sectores urbanos
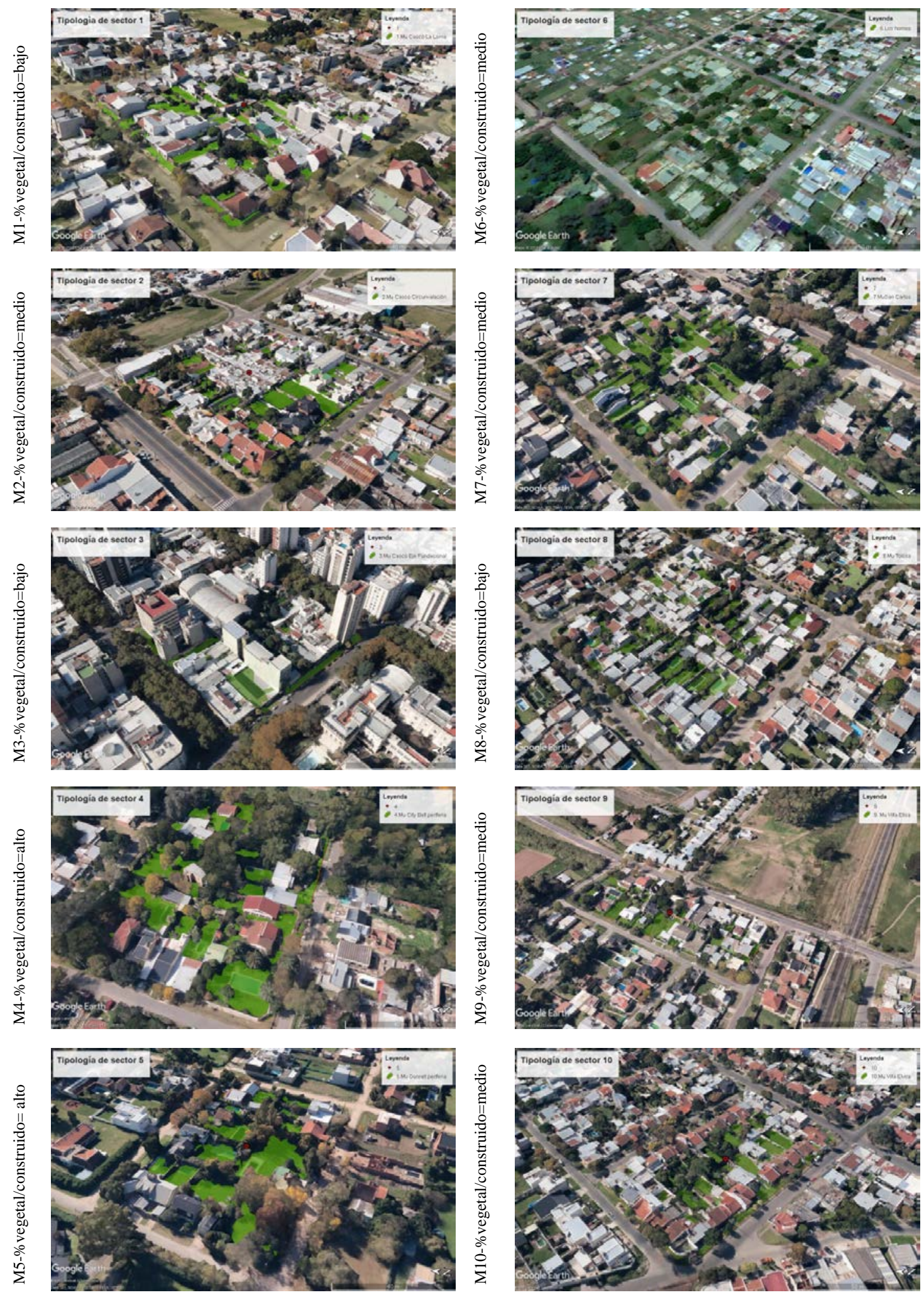


\section{Método de cálculo de $\mathrm{GD}_{\text {mes }}$ con datos oficiales y experimentales}

A partir de los datos climáticos oficiales y de los experimentales, se calcularon los $\mathrm{GD}_{\text {mes. }}$ Para el cálculo de los GD en función de datos climáticos oficiales - con base en temperaturas medias mensuales -, se utilizan las Ecuaciones 1 y 2 (DEGREE..., 2007) presentadas a continuación:

$\mathrm{GD}_{\text {cal }}=\left[\mathrm{T}_{\text {base }}-\left(\mathrm{T}_{\text {máx }}+\mathrm{T}_{\text {mín }}\right) / 2\right] \times \mathrm{n}^{\mathrm{o}}$ días $\times 1$ Ec. 1

$\mathrm{GD}_{\text {ref }}=\left[\left(\mathrm{T}_{\text {máx med }}-\mathrm{T}_{\text {base }}\right) / 2\right] \times \mathrm{n}^{\mathrm{o}}$ días $\times 1 \quad$ Ec. 2

Donde:

$\mathrm{T}_{\text {base }}=$ temperatura de base $\left(18^{\circ} \mathrm{C}\right.$ en invierno; 23

${ }^{\circ} \mathrm{C}$ en verano);

$\mathrm{T}_{\text {máx }}=$ temperatura máxima absoluta $\left({ }^{\circ} \mathrm{C}\right)$;

$\mathrm{T}_{\text {mín }}=$ temperatura mínima absoluta $\left({ }^{\circ} \mathrm{C}\right) ; \mathrm{y}$

$\mathrm{T}_{\text {máx med }}=$ temperatura máxima media $\left({ }^{\circ} \mathrm{C}\right)$.

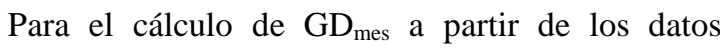
experimentales, se consideran las temperaturas diarias máxima y mínima absolutas del aire exterior ( $\mathrm{T}_{\text {máx }}$ y $\left.\mathrm{T}_{\text {mín }}\right)$ y una temperatura base determinada para el confort del habitante (estimación de la temperatura del aire exterior en la que no se requiere climatización artificial). Las condicionantes de cálculo se muestran en Tabla 2.

Una vez calculados los GD, se debe georreferenciarlos. A partir de las mediciones discretas, se aplican técnicas geoestadísticas para construir mapas con variables continuas. En este caso, se adopta el método de ponderación inversa a la distancia (IDW) y se obtienen mapas Raster con los GD para invierno y verano.

\section{Identificación de zonas de similar comportamiento térmico}

A los efectos de interpretar los resultados y de profundizar el análisis, se realiza un agrupamiento de los distintos puntos de medición a partir de la técnica de clustering de k-medias según su afinidad en el comportamiento térmico (PÉREZ LÓPEZ, 2004; CHÉVEZ et al., 2017). Para ello se adopta una serie de 32 días (16 de verano y 16 de invierno), la cual presenta la temperatura mínima y máxima para cada día. Por ende, cada punto de medición cuenta con un vector asociado de 64 valores normalizados entre 0 y 1 . Dichos vectores se agrupan entre sí en función de su similitud, lo cual permite detectar grandes áreas urbanas con comportamientos térmicos característicos y, de esta

${ }^{4} \mathrm{Si}$ bien el cálculo de la demanda de refrigeración para verano generalmente considera distintos aspectos (carga por conducción, ventilación, radiación solar y por fuentes internas), manera, analizar sus vínculos con los diferentes grados de consolidación urbana.

\section{Estimación de las demandas de energía teórica a partir de GD oficiales y experimentales}

Para cada MU se realiza el cálculo de la demanda de energía teórica para climatización, con base en la morfología del sector, teniendo en cuenta:

(a) la información térmica exterior obtenida de una de las estaciones meteorológicas oficiales; y

(b) la información térmica exterior obtenida de forma experimental.

Posteriormente, es posible comparar la demanda teórica 'oficial' y 'experimental' de cada MU e identificar las diferencias obtenidas, lo cual permite minimizar los errores de cálculo. Para ello se calcula un coeficiente volumétrico de pérdidas de calor por mosaico $\left(\mathrm{G}_{\mathrm{MU}}\right)$ en base a coeficientes medios de transmitancia térmica $\left(\mathrm{K}_{\text {medio }}\right)$ de los elementos que componen la envolvente - techos, pisos, muros y ventanas -, los cuales se consideran constantes en los diferentes MU. A partir del $\mathrm{G}_{\mathrm{MU}} \mathrm{y}$ de los GD $\mathrm{GD}_{\text {mes }}$ promedio de calefacción y refrigeración, se calcula la demanda de energía en base $18{ }^{\circ} \mathrm{C}$ para invierno y $23{ }^{\circ} \mathrm{C}$ para verano ${ }^{4} \mathrm{a}$ partir de la Ecuación 3. Esta ecuación es un cálculo validado de estimación de la demanda de energía para edificios individuales, desarrollada en normas argentinas no obligatorias certificadas por un organismo oficial (INSTITUTO..., 1996a, 1996b):

$\mathrm{Q}(\mathrm{kWh})=\left(\mathrm{T} \times \mathrm{G}_{\mathrm{MU}} \times \mathrm{GD}_{\text {mes }} \times \mathrm{V}\right) / 1000 \quad$ Ec. 3

Donde:

$\mathrm{T}$ = tiempo de calefacción (24h);

$\mathrm{G}_{\mathrm{MU}}=$ coeficiente volumétrico de pérdidas;

$\mathrm{GD}_{\text {mes }}=$ grados día mensuales; $\mathrm{y}$

$\mathrm{V}=$ volumen construido de MU.

Los relevamientos urbanos parciales de cada MU y las características edilicias relevados oportunamente en proyectos de investigación previos en la totalidad del área de estudio (INSTITUTO..., 1987; ROSENFELD, 1999) permiten adoptar las siguientes consideraciones con respecto a los coeficientes de transmitancia térmica ( $\mathrm{K}_{\text {medios }}$ ). Se establece que el $50 \%$ de las viviendas cuenta con una calidad de edificación insuficiente y que el otro $50 \%$ presenta una calidad básica y satisfactoria. Respecto al área vidriada, se considera una relación entre la superficie de ventanas y la

ha sido simplificado a los efectos de cálculo para utilizar los GDref. Se consideró, entonces, un cálculo análogo al de Qcal. 
superficie construida del 15\%. En consecuencia, se adoptan los valores de $\mathrm{K}_{\text {medio }}$ expresados en la Tabla 3.

En función de los valores de $\mathrm{G}$ de cada uno de los MU y de los valores de GD oficiales y experimentales, se calculó la demanda de energía para calefacción y refrigeración, con lo cual se puede identificar las diferencias entre valores de ambas aproximaciones y estimar con mayor precisión la demanda de los distintos sectores urbanos. Esto permite orientar las medidas de mejoramiento energético a aquellos sectores donde los consumos sean críticos.

\section{Resultados y discusión}

Una vez descripta la metodología, se realiza el análisis propuesto y se presentan los principales resultados y la discusión.

\section{Análisis de las temperaturas externas de los MU}

En la Figura 3 se muestran las temperaturas registradas en junio/julio de 2012 (invierno) y en el mes de enero de 2012 (verano).
Se pueden observar amplias diferencias en los distintos sectores urbanos.

Las temperaturas medias de invierno oscilan entre $9,3{ }^{\circ} \mathrm{C}$ y $14,3{ }^{\circ} \mathrm{C}$, con lo cual se registra una diferencia - o amplitud térmica - entre los extremos de $5{ }^{\circ} \mathrm{C}$. La temperatura promedio más baja corresponde a la periferia Oeste de La Plata (MU3), y la más elevada, a la zona central inserta en el CF (MU1). Más allá de estos promedios, se pueden observar amplias diferencias en las temperaturas mínimas absolutas, ya que áreas de la periferia Norte, Sur y Oeste registran valores muy bajos cercanos a $-1,5^{\circ} \mathrm{C}$, en relación a sectores centrales con $8,2{ }^{\circ} \mathrm{C}$ de temperatura mínima. Con relación a estos datos, los mosaicos que presentan muy bajas temperaturas mínimas también registran temperaturas máximas mayores, correspondiendo a sectores con mayor amplitud térmica que el resto. Estos sectores son el MU6 (Los Hornos), el MU9 (Villa Elisa) y el MU10 (Villa Elvira).

\section{Tabla 2 - Cálculo de los GD}

\begin{tabular}{|c|c|c|c|}
\hline \multicolumn{2}{|c|}{ GD de calefacción } & \multicolumn{2}{|c|}{ GD de enfriamiento } \\
\hline Condición & Fórmula usada & Condición & Fórmula usada \\
\hline $\mathrm{T}_{\text {mín }}>\mathrm{T}_{\text {base }}$ & $\mathrm{D}_{\mathrm{h}}=0$ & $\mathrm{~T}_{\text {máx }}<\mathrm{T}_{\text {base }}$ & $\mathrm{D}_{\mathrm{c}}=0$ \\
\hline$\left(\mathrm{T}_{\text {máx }}+\mathrm{T}_{\text {mín }}\right) / 2>\mathrm{T}_{\text {base }}$ & $\mathrm{D}_{\mathrm{h}}=\left(\mathrm{T}_{\text {base }}-\mathrm{T}_{\text {mín }}\right) / 4$ & $\left(\mathrm{~T}_{\text {máx }}+\mathrm{T}_{\text {mín }}\right) / 2<\mathrm{T}_{\text {base }}$ & $\mathrm{D}_{\mathrm{c}}=\left(\mathrm{T}_{\text {máx }}-\mathrm{T}_{\text {base }}\right) / 4$ \\
\hline $\mathrm{T}_{\text {máx }}>=\mathrm{T}_{\text {base }}$ & $\begin{array}{l}\mathrm{D}_{\mathrm{h}}=\left[\left(\mathrm{T}_{\text {base }}-\mathrm{T}_{\text {mín }}\right) / 2\right]- \\
{\left[\left(\mathrm{T}_{\text {máx }}-\mathrm{T}_{\text {base }}\right) / 4\right]}\end{array}$ & $\mathrm{T}_{\min } \leq \mathrm{T}_{\text {base }}$ & $\begin{array}{l}\mathrm{D}_{\mathrm{c}}=\left[\left(\mathrm{T}_{\text {máx }}-\mathrm{T}_{\text {base }}\right) / 2\right]- \\
{\left[\left(\mathrm{T}_{\text {base }}-\mathrm{T}_{\text {mín }}\right) / 4\right]}\end{array}$ \\
\hline $\mathrm{T}_{\text {máx }}<\mathrm{T}_{\text {base }}$ & $D_{h}=T_{\text {base }}-\left[\left(T_{\text {máx }}+T_{\text {mín }}\right) / 2\right]$ & $\mathrm{T}_{\min }>\mathrm{T}_{\text {base }}$ & $\mathrm{D}_{\mathrm{c}}=\left(\mathrm{T}_{\text {máx }}+\mathrm{T}_{\text {mín }}\right) / 2-\mathrm{T}_{\text {bas }}$ \\
\hline
\end{tabular}

Tabla 3 - Transmitancia térmica promedio de la envolvente de los MU de La Plata

\begin{tabular}{|c|c|c|}
\hline Techos & 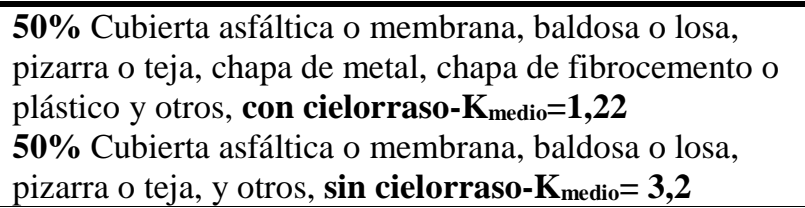 & $\mathrm{K}_{\text {medio }}=2,21 \mathrm{~W} / \mathrm{m}^{2}{ }^{\circ} \mathrm{C}$ \\
\hline Pisos & Cerámica/ cemento o ladrillo fijo. & $\mathrm{K}_{\text {medio }}=1,57 \mathrm{~W} / \mathrm{m}^{2}{ }^{\circ} \mathrm{C}$ \\
\hline Muros & 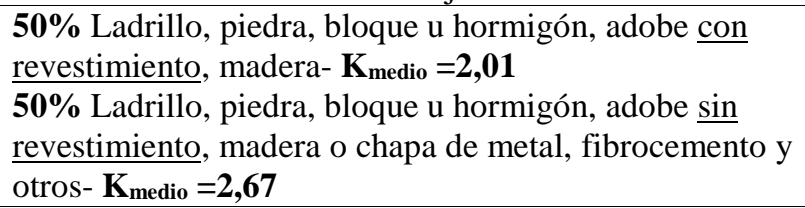 & $\mathrm{K}_{\text {medio }}=2 \mathrm{~W} / \mathrm{m}^{2}{ }^{\circ} \mathrm{C}$ \\
\hline Vidriados & Aberturas de vidrio simple (100\% de las tipologías) & $\mathrm{K}_{\text {medio }}=5,8 \mathrm{~W} / \mathrm{m}^{2}{ }^{\circ} \mathrm{C}$ \\
\hline
\end{tabular}


Figura 3 - Registros de temperatura obtenidos en los MU para junio/julio (a) y enero de 2012 (b)

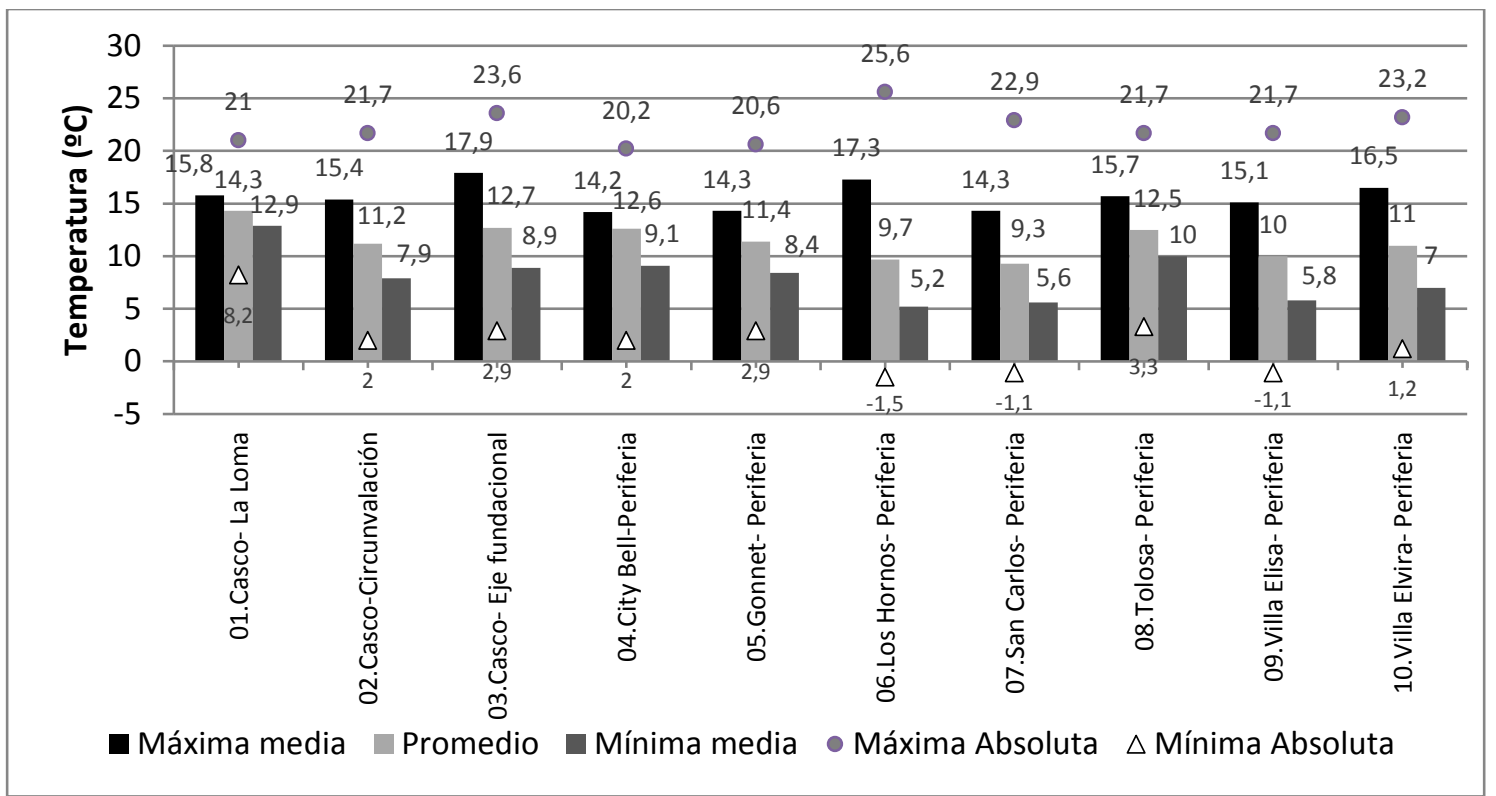

(a)

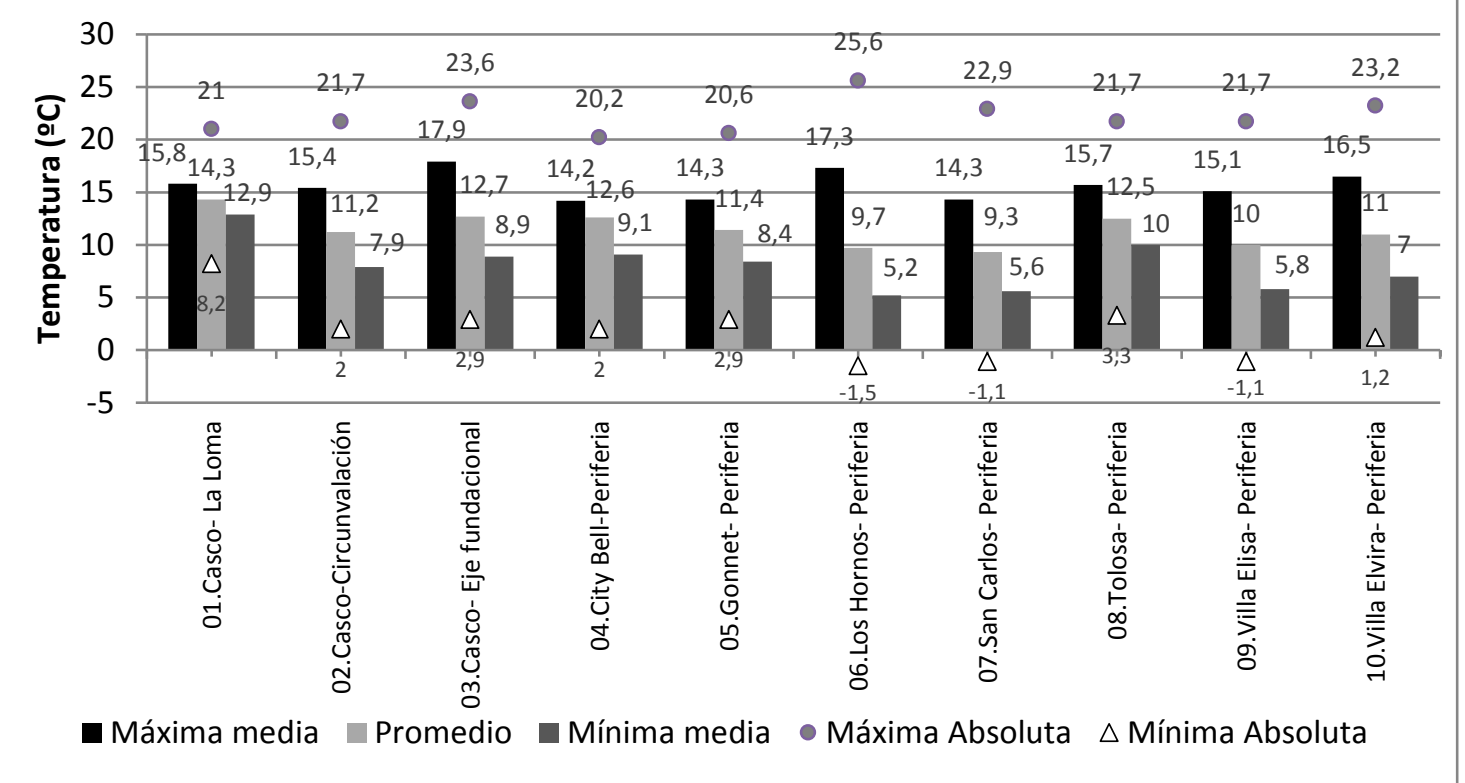

(b)

Asimismo, se observan variaciones en las temperaturas medias de verano, que oscilan entre $24,3{ }^{\circ} \mathrm{C}$ y $27,6^{\circ} \mathrm{C}$ (una diferencia de más de $3^{\circ} \mathrm{C}$ ), siendo el valor mínimo registrado en la periferia (MU7) y el máximo registrado en el CF de la ciudad (MU1). El análisis de las temperaturas mínimas y máximas medias muestra que existen sectores con grandes amplitudes térmicas que responden a bajas temperaturas nocturnas y altas temperaturas diurnas. Estos sectores son el MU6 (Los Hornos), el MU9 (Villa Elisa) y el MU7 (San Carlos), localizados en los límites urbanos de la ciudad, cercanos a áreas rurales, expuestos a os vientos. En cambio, en la zona céntrica del CF (MU1, MU2 y
MU3), se observan temperaturas muy estables entre máximas y mínimas medias, lo cual puede explicarse por la mayor masa edilicia existente, ocasionando un efecto de amortiguamiento térmico y de acumulación de calor. Estas características generan temperaturas mínimas más elevadas, mientras que las temperaturas máximas atenúan sus picos.

Finalmente, se observa cierta correlación entre los dos períodos analizados (invierno/verano) ya que existe gran amplitud térmica en áreas cercanas al ámbito rural y estabilidad térmica en zonas centrales más consolidadas. 
En la Tabla 4 se muestran los resultados de las temperaturas medias de invierno y de verano, junto con las características morfológicas de cada sector: superficie del terreno, superficie ocupada, superficie construida, factor de ocupación total $\left(\mathrm{FOT}^{5}\right)$, factor de ocupación del suelo $\left(\mathrm{FOS}^{6}\right.$ ), relación de la superficie vegetal versus la superficie construida y arbolado urbano. Las variables en cada MU fueron relevadas a partir del trabajo de campo y observación fotográfica.

Se presupone que, en invierno, las áreas de menor temperatura deberían tener menores valores de FOS y de FOT y que, en verano, las temperaturas deberían ser mayores en áreas con mayor FOS y FOT. Si bien no se observa una relación directa entre el FOS, el FOT y las temperaturas medias, se puede verificar que los sectores con menores temperaturas en invierno y mayor temperatura en verano (sectores 06.Los Hornos, 07.San Carlos, 09.Villa Elisa y 10.Villa Elvira) se localizan cercanos a áreas abiertas rurales (MU6 y MU7) o de bañados (MU9 y MU10), lugares que presentan menor densidad edilicia y que tienen una relación vegetal/construido media. Mientras que los de mayor temperatura en invierno se localizan en zonas mediterráneas del área urbana (sectores 08, 04, 03, 01), ya sea en el CF o en nuevas centralidades (City Bell, Tolosa y Gonnet), con condiciones vegetal/construido que varían entre alta, media y baja.

Podemos decir, entonces, que tanto los coeficientes FOS y FOT como la vegetación no son capaces de explicar por sí solos las condiciones micro climáticas de los sectores urbanos, ya que la situación responde a una combinación de factores tales como la incidencia de los vientos, los tipos de superficie absorbente, la morfología, entre otros, los cuales serán incorporados en futuros análisis.

\section{Resultados del cálculo de los GDmes en los MU y estaciones oficiales}

La Tabla 5 muestra los valores de los GD mensuales y anuales calculados con información oficial del OALP y del ALP, a partir de las temperaturas medias del año 2012 y del cálculo para la estación ALP a partir de la serie histórica.

Tabla 4 - Relación entre temperaturas medias y morfología urbana

\begin{tabular}{|c|c|c|c|c|c|c|c|c|c|c|}
\hline$\stackrel{2}{\Sigma}$ & 足 & 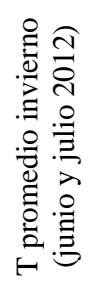 &  & 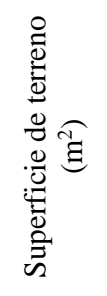 &  & 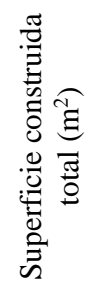 & ర్ & $\bigcup_{1}^{\infty}$ &  & 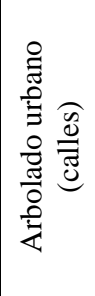 \\
\hline $\begin{array}{l}01 . \text { Casco- } \\
\text { La Loma }\end{array}$ & $\begin{array}{c}\text { Urbana/ } \\
\text { mediterránea }\end{array}$ & 14,3 & 27,6 & 14690 & 6630 & 8997 & 0,6 & 0,5 & Bajo & Medio \\
\hline $\begin{array}{l}\text { 02.Casco- } \\
\text { Circunvalación }\end{array}$ & $\begin{array}{c}\text { Urbana/ } \\
\text { mediterránea }\end{array}$ & 11,2 & 26,8 & 14387 & 6970 & 2743 & 0,2 & 0,5 & Medio & Bajo \\
\hline $\begin{array}{l}\text { 03.Casco- } \\
\text { Eje fundacional }\end{array}$ & $\begin{array}{c}\text { Urbana/ } \\
\text { mediterránea }\end{array}$ & 12,7 & 25,8 & 14518 & 8863 & 17138 & 1,2 & 0,6 & Bajo & Alto \\
\hline $\begin{array}{l}\text { 04.City Bell- } \\
\text { Periferia }\end{array}$ & Urbana/dispersa & 12,6 & 25,1 & 10920 & 1895 & 1601 & 0,1 & 0,2 & Alto & Alto \\
\hline $\begin{array}{l}\text { 05.Gonnet- } \\
\text { Periferia }\end{array}$ & Urbana/dispersa & 11,4 & 26,0 & 8433 & 1835 & 5317 & 0,6 & 0,2 & Alto & Alto \\
\hline $\begin{array}{l}\text { 06.Los Hornos- } \\
\text { Periferia }\end{array}$ & Abierta/rural & 9,7 & 25,4 & 7937 & 1324 & 8587 & 1,1 & 0,2 & Medio & Bajo \\
\hline $\begin{array}{l}\text { 07.San Carlos- } \\
\text { Periferia }\end{array}$ & Abierta/rural & 9,3 & 24,3 & 14335 & 4690 & 4895 & 0,3 & 0,3 & Medio & Alto \\
\hline $\begin{array}{l}\text { 08.Tolosa- } \\
\text { Periferia }\end{array}$ & $\begin{array}{c}\text { Urbana/ } \\
\text { mediterránea }\end{array}$ & 12,5 & 26,4 & 13537 & 7113 & 2420 & 0,2 & 0,5 & Alto & Bajo \\
\hline $\begin{array}{l}\text { 09.Villa Elisa- } \\
\text { Periferia }\end{array}$ & Abierta/rural & 10,0 & 25,8 & 9601 & 3613 & 4851 & 0,5 & 0,4 & Medio & Bajo \\
\hline $\begin{array}{l}\text { 10.Villa Elvira- } \\
\text { Periferia }\end{array}$ & Abierta/rural & 11,0 & 25,5 & 9815 & 4337 & 8664 & 0,9 & 0,4 & Medio & Bajo \\
\hline
\end{tabular}

${ }^{5}$ Porcentaje de superficie útil de una parcela que se puede ocupar con los usos establecidos; el resto será destinado a espacio libre. Cada sector urbano posee un indicador límite según norma. FOS=Sup. cub. planta baja / Sup. de la parcela.
${ }^{6}$ Coeficiente que, multiplicado por la superficie total de la parcela, da como resultado la superficie total máxima edificable. Cada sector urbano posee un indicador límite según norma. FOT = Sup. cubierta total / Sup. de la parcela. 
Tabla 5 - GD cal y GD ref de acuerdo con las informaciones del OALP7 (año 2012), del ALP (año 2012) y del ALP (serie 1980/2009)

\begin{tabular}{|c|c|c|c|c|c|c|c|c|c|c|}
\hline Mes/Año & \begin{tabular}{|l|} 
número \\
de días
\end{tabular} & $\begin{array}{c}\text { T med. } \\
{ }^{\circ} \mathrm{C}\end{array}$ & $\begin{array}{l}\text { T max. } \\
\text { Med. }{ }^{\circ} \mathrm{C}\end{array}$ & GD & $\begin{array}{c}\text { T med. } \\
{ }^{\circ} \mathrm{C}\end{array}$ & $\begin{array}{l}\mathrm{T} \text { max. } \\
\text { med. }{ }^{\circ} \mathrm{C}\end{array}$ & GD & $\mathrm{T}$ med. ${ }^{\circ} \mathrm{C}$ & $\begin{array}{c}\text { T } \\
\text { max. } \text { med. }{ }^{\circ} \mathrm{C}\end{array}$ & GD \\
\hline Enero & 31 & 24,2 & 29,7 & 103 & 26,1 & 31,7 & 135 & 22,4 & 28,6 & 87 \\
\hline Febrero & 28 & 23,6 & 30,4 & 104 & 25,1 & 29,4 & 90 & 21,9 & 28,2 & 73 \\
\hline Marzo & 31 & 20,5 & 25,4 & 37 & 21,5 & 25,7 & 42 & 19,5 & 25,5 & 39 \\
\hline Mayo & 31 & 15,3 & 15,7 & 84 & 16,1 & 20,1 & 59 & 13 & 18,4 & 155 \\
\hline Junio & 30 & 10,4 & 14,3 & 228 & 11,1 & 16,5 & 207 & 9,8 & 14,7 & 246 \\
\hline Julio & 31 & 7,9 & 13,6 & 313 & 8,5 & 13,6 & 295 & 9,2 & 14,4 & 273 \\
\hline Agosto & 31 & 12,3 & 18,0 & 177 & 12,8 & 16,7 & 161 & 10,2 & 15,8 & 242 \\
\hline Septiembre & 30 & 14,1 & 17,8 & 117 & 15 & 19,5 & 90 & 12,4 & 17,9 & 168 \\
\hline Octubre & 31 & 16,9 & 20,9 & 34 & 17,8 & 21,7 & 6 & 14,7 & 20,3 & 102 \\
\hline GD ref & & & & 383 & & & 415 & & & 274 \\
\hline
\end{tabular}

Se observan diferencias entre las dos localizaciones analizadas. En invierno, el OALP (localización urbana) presentó mayor cantidad de GD de calefacción anual (998 GD $\mathrm{Gal}_{\mathrm{cal}}$ ) en relación a los registros de la estación ALP (localización rural) de ese año (836 GD cal $_{\text {ca }}$. Sin embargo, el OALP tuvo menor $\mathrm{GD}_{\text {cal }}$ respecto de la serie climática del ALP (998 versus $1246 \mathrm{GD}_{\text {cal, }}$ respectivamente). Es evidente que los registros del OALP frente a los del ALP están influenciados por su entorno urbano, elevándose la temperatura debido a la carga y a la inercia térmica que proporciona la ciudad y la forestación circundante.

Por lo tanto, se considera para el caso de invierno del año 2012 una situación promedio para los meses de junio y julio que presenta los siguientes valores: $\left[\right.$ OALP $(2012)=270$ GD $\left._{\text {cal }}\right],[$ ALP $(2012)=251$ $\left.\mathrm{GD}_{\text {cal }}\right]$ y $\left[\mathrm{ALP}\right.$ (serie climática) $\left.=259 \mathrm{GD}_{\text {cal }}\right]$.

En cuanto a la condición climática de verano, los registros de la serie climática de ALP presentaron un valor menor en cuanto a los $\mathrm{GD}_{\text {ref }}$ anual (274 $\mathrm{GD}_{\text {ref }}$ ) en relación a los registrados en el año 2012, tanto en el OALP (383 GD ref) como en el ALP (415 $\mathrm{GD}_{\text {ref }}$ ).

En el caso del mes de enero, considerado para la comparativa de verano con los puntos de medición, se obtuvieron los siguientes resultados: [OALP $\left.(2012)=103 G_{\text {ref }}\right],\left[\right.$ ALP $\left.(2012)=135 G_{\text {ref }}\right] \mathrm{y}$ [ALP $($ serie climática $)=87$ GD $\left._{\text {ref }}\right]$.

En síntesis, se observa que el año 2012 fue extremo, tanto en el invierno como en el verano. Estas consideraciones son importantes a los efectos de obtener conclusiones con respecto a los registros experimentales realizados.

Para realizar un análisis detallado de los GD en los distintos MU de La Plata, se aplica la fórmula enunciada en Tabla 4, presentada anteriormente. La Figura 4 muestra los resultados obtenidos.

Por su parte, la Tabla 6 muestra la relación entre los GD y las variables morfológicas y vegetales de cada zona.

El cálculo demuestra que en invierno hay algunos MU que registran prácticamente un $100 \%$ más de GD que otros, como es el caso de zonas de la periferia urbana (04.City Bell; 05.Gonnet; 06.Los Hornos; 07.San Carlos; 09.Villa Elisa) en relación al CF (01.La Loma y 03.Casco eje), de acuerdo con la Tabla 4. Con relación a este aspecto, los resultados de Correa, De Rosa y Lesino (2008) muestran una sobrestimación en la ciudad de Mendoza del 50\%.

7Información suministrada por el Departamento de Sismología e Información Meteorológica, de la Facultad de Ciencias Astronómicas y Geofísicas (FCAG) de la Universidad Nacional de La Plata (UNLP). 
Figura 4 - GD experimentales. En invierno se calculan 31 días $\left(\mathrm{T}_{\text {base }}=18^{\circ} \mathrm{C}\right)$ - en verano se calculan 31 días $\left(\mathrm{T}_{\text {base }}=23^{\circ} \mathrm{C}\right)$ - aclaración: el MU2 registró un período menor en verano, con lo cual se realizó una inferencia estadística para estimar el valor mensual

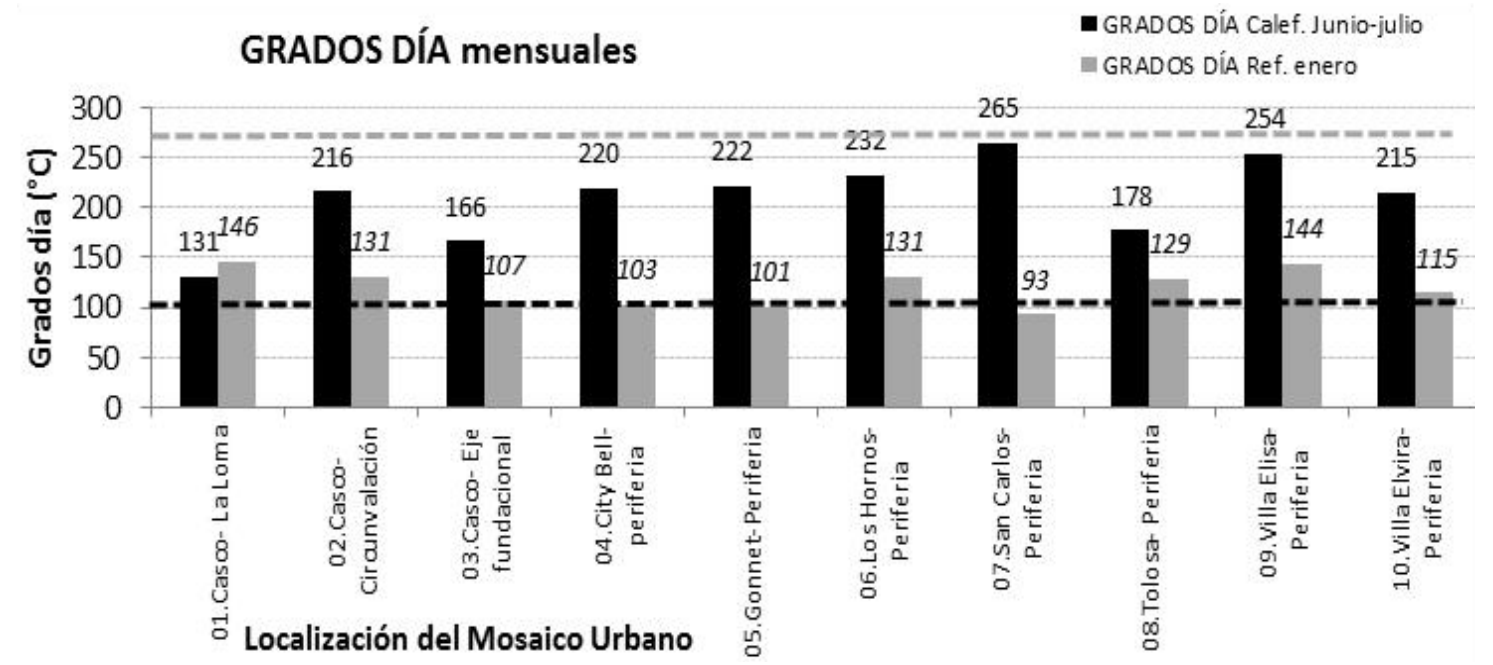

Tabla 6 - Relación entre los GD y las características morfológicas y vegetadas de cada sector urbano, ordenadas en forma ascendente de los GD de invierno y en forma descendente los GD de verano

\begin{tabular}{|c|c|c|c|c|c|c|c|c|c|c|}
\hline$\stackrel{P}{\Sigma}$ & & 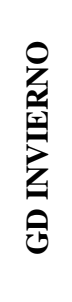 & 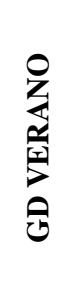 & 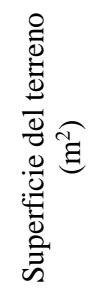 & 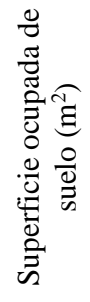 & 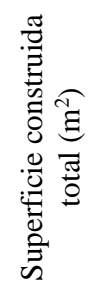 & 包 & $\underset{\mathscr{O}}{\mathscr{O}}$ &  & 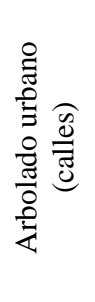 \\
\hline $\begin{array}{l}\text { 01.Casco- } \\
\text { La Loma }\end{array}$ & $\begin{array}{c}\text { Urbana/ } \\
\text { mediterránea }\end{array}$ & 131 & 146 & 14690 & 6630 & 8997 & 0,6 & 0,5 & Bajo & Medio \\
\hline $\begin{array}{l}\text { 03.Casco- } \\
\text { Eje fundacional }\end{array}$ & $\begin{array}{c}\text { Urbana/ } \\
\text { mediterránea }\end{array}$ & 166 & 107 & 14518 & 8863 & 17138 & 1,2 & 0,6 & Bajo & Alto \\
\hline $\begin{array}{l}\text { 08.Tolosa- } \\
\text { Periferia }\end{array}$ & $\begin{array}{c}\text { Urbana/ } \\
\text { mediterránea }\end{array}$ & 178 & 129 & 13537 & 7113 & 2420 & 0,2 & 0,5 & Alto & Bajo \\
\hline $\begin{array}{l}\text { 10.Villa Elvira- } \\
\text { Periferia }\end{array}$ & Abierta/rural & 215 & 115 & 9815 & 4337 & 8664 & 0,9 & 0,4 & Medio & Bajo \\
\hline $\begin{array}{l}\text { 02.Casco- } \\
\text { Circunvalación }\end{array}$ & $\begin{array}{c}\text { Urbana/ } \\
\text { mediterránea }\end{array}$ & 216 & 131 & 14387 & 6970 & 2743 & 0,2 & 0,5 & Medio & Bajo \\
\hline $\begin{array}{l}\text { 04.City Bell- } \\
\text { periferia }\end{array}$ & Urbana/dispersa & 220 & 103 & 10920 & 1895 & 1601 & 0,1 & 0,2 & Alto & Alto \\
\hline $\begin{array}{l}\text { 05.Gonnet- } \\
\text { Periferia }\end{array}$ & Urbana/dispersa & 222 & 101 & 8433 & 1835 & 5317 & 0,6 & 0,2 & Alto & Alto \\
\hline $\begin{array}{l}\text { 06.Los Hornos- } \\
\text { Periferia }\end{array}$ & Abierta/rural & 232 & 131 & 7937 & 1324 & 8587 & 1,1 & 0,2 & Medio & Bajo \\
\hline $\begin{array}{l}\text { 09.Villa Elisa- } \\
\text { Periferia }\end{array}$ & Abierta/rural & 254 & 144 & 9601 & 3613 & 4851 & 0,5 & 0,4 & Medio & Bajo \\
\hline $\begin{array}{l}\text { 07.San Carlos- } \\
\text { Periferia }\end{array}$ & Abierta/rural & 265 & 93 & 14335 & 4690 & 4895 & 0,3 & 0,3 & Medio & Alto \\
\hline
\end{tabular}

Ya en lo que refiere a las condiciones del verano, la situación cambia. Si bien existen diferencias entre los distintos puntos de la ciudad, éstas no son tan pronunciadas como en el invierno. Se observan registros cercanos a los $100 \mathrm{GD}_{\text {ref }}$ y puntos más cálidos con registros máximos como el caso del centro de la ciudad (MU1) con 146 GD $_{\text {ref, }}$ lo que representa una diferencia de aproximadamente un $50 \%$. Si los comparamos con los datos de la serie climática histórica, que registra $87 \mathrm{GD}_{\text {ref, }}$ podemos observar que los registros experimentales resultaron siempre mayores, con lo cual se estaría subestimando su incidencia en el confort térmico. Sin embargo, respecto del año 2012, en el OALP se 
aprecia que, siendo un año más cálido a nivel general, con $103 \mathrm{GD}_{\text {ref }}$ en enero, los registros experimentales fueron mayores en tres puntos claves de la ciudad (ver Tabla 6); estos fueron el centro urbano (01.Casco La Loma, 146 GD $_{\text {ref }}$ ) y las periferias lindantes con áreas agrícolas o descampadas como (06.Los Hornos, 131 GD $_{\text {ref }}$ ) y (10.Villa Elisa, $\left.144 \mathrm{GD}_{\text {ref }}\right)$. Con esto se concluye que los sectores periféricos y del CF pueden ser tomados como casos clave para tener en cuenta sus condiciones térmicas externas diferenciadas y extremas, con lo cual ajustar el cálculo de demanda de energía para climatización. A modo comparativo, los cálculos de subestimación de GD con base en datos experimentales en la ciudad de Mendoza obtenidos por Correa, De Rosa y Lesino (2008) fueron del 9\%.

En cuanto a los registros de GD mensuales de calefacción y refrigeración, los mismos pueden ser explicados a partir del nivel de consolidación urbana (descripta en la metodología) que puede observarse en la Figura 5.

Los menores requerimientos $\mathrm{GD}_{\text {cal }}$ de invierno se detectaron en sectores de consolidación alta y media, que son sectores con una densidad habitacional y poblacional elevada. Luego se verificaron valores intermedios de $\mathrm{GD}_{\text {cal }}$ en los sectores de consolidación media, con menor densidad de viviendas y de población (menor carga e inercia térmica). Finalmente, los mayores valores de $\mathrm{GD}_{\text {cal }}$ responden a sectores con consolidaciones medias y bajas y con menores densidades de vivienda y de habitantes; se trata de áreas dispersas y cubiertas con más vegetación (pisos absorbentes y arbolados).

En el caso del verano, los valores más altos de $\mathrm{GD}_{\text {ref }}$ se registraron en áreas de consolidación alta y baja, con densidad poblacional y habitacional elevada y baja, respectivamente. Mientras que los valores intermedios se registraron en áreas de consolidación media, coincidiendo la descripción realizada en el párrafo anterior (ver Figura 5).

A los efectos de establecer una correlación entre lo registrado en invierno y verano, la Figura 5 muestra una zonificación a partir de la interpolación de los GD calculados.

En invierno se observa una distribución homogénea, con menores requerimientos de $\mathrm{GD}_{\text {cal }}$ en sectores de alta consolidación, correspondientes al CF y sectores consolidados de la periferia (microcentros), mientras que se observan mayores valores de $\mathrm{GD}_{\mathrm{cal}}$ en áreas periféricas dispersas.

La distribución en verano parece ser más compleja, ya que se observan tres puntos más cálidos (mayores $\mathrm{GD}_{\mathrm{ref}}$ ) y, entre ellos, espacios intersticiales más frescos con menores $\mathrm{GD}_{\text {ref. }}$ Esta particularidad conlleva a analizar - en la siguiente sección - las curvas de temperatura de los distintos sectores de la ciudad en verano e invierno, para intentar alcanzar un mayor grado de comprensión del fenómeno.

\section{Figura 5 - $\mathbf{G D}_{\text {cal }}$ y $\mathbf{G D}_{\text {ref }}$ y nivel de consolidación urbana}




Figura 6 - Zonificación de GD $_{\text {cal }}$ (izquierda) y GD ref (derecha) de la ciudad de La Plata

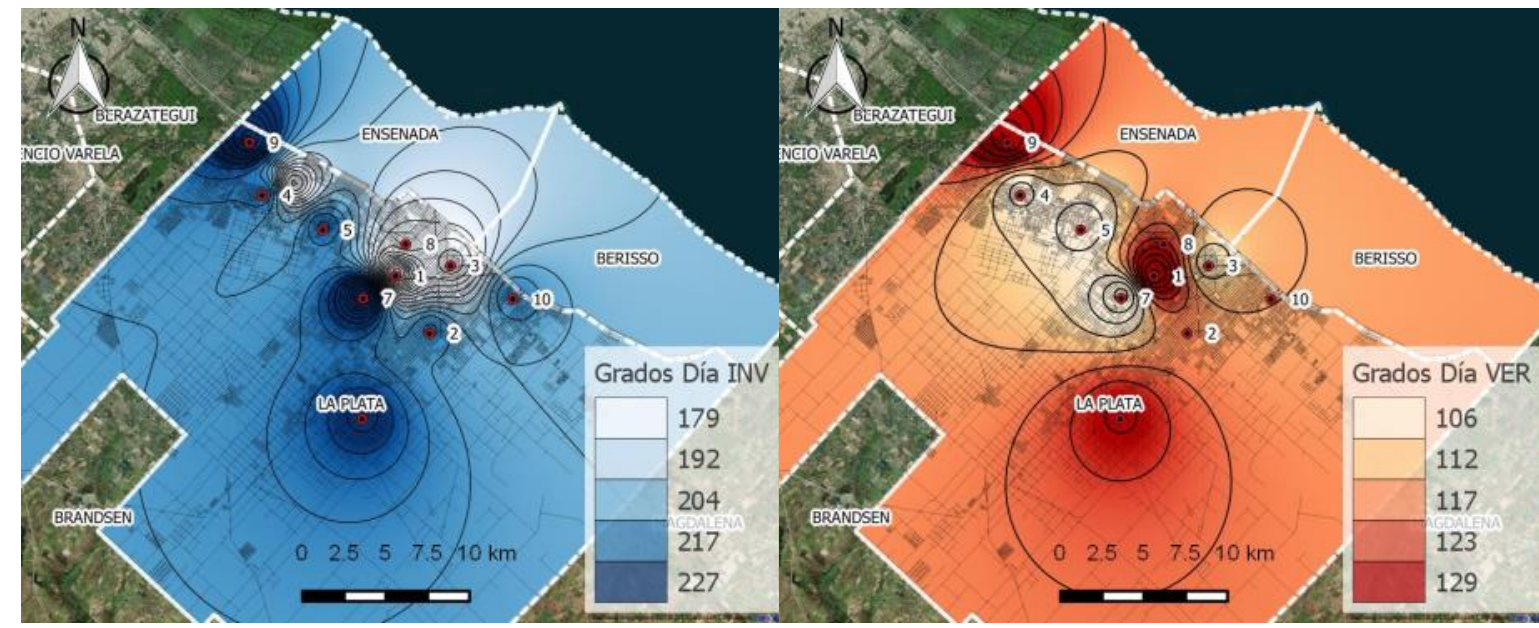

\section{Análisis de zonas de comportamiento térmico similar}

A partir del análisis surgido de los mapas de la Figura 6, se buscó agrupar entre sí los distintos sectores urbanos en función de su comportamiento térmico de invierno y de verano a partir de la técnica de clustering de k-medias. Como resultado se identificaron tres grupos y se calculó una curva promedio de temperaturas que representara a cada uno de ellos (ver Figura 7). El grupo 1 contiene un único sector urbano, localizado en el centro de la ciudad y que presenta una consolidación alta (MU1). En el grupo 2 se encuentran las áreas que principalmente son de consolidación media (MU2, MU3, MU4, MU5, MU8 y MU10). Finalmente, en el grupo 3 se encuentran los sectores de consolidación baja, lindantes con áreas rurales o de bañados (MU6, MU7 y MU9).

Se observa que los tres grupos son claramente diferenciables, sobre todo al observar las temperaturas mínimas. En invierno las tres curvas se separan, advirtiendo claramente que el grupo 1 cuenta con GD menores; que el grupo 2 presenta un valor intermedio de GD; y que el grupo 3 cuenta con los valores máximos de GD. Sin embargo, en verano las curvas se entrecruzan. Se observa que algunos sectores de la periferia - que se suponen más frescos durante el día - tienen como singularidad que, si bien se reduce la temperatura nocturna (aprovechamiento de refrescamiento selectivo nocturno), se registran temperaturas elevadas durante el día. Se verifica una mayor amplitud térmica, con lo cual el cálculo de $\mathrm{GD}_{\text {ref }}$ brinda resultados similares a otros lugares más estables (de menor amplitud térmica), encubriendo la necesidad de mayores demandas para climatización en estas áreas. Es el caso de las regiones centrales de la ciudad, en donde se observó estabilidad térmica debido a la masa construida. Las temperaturas son elevadas durante la noche y también durante el día. Allí también el cálculo de GD es alto. Entre estos dos casos extremos, se encuentra la mayoría de los puntos urbanos medidos, con situaciones intermedias.

En conclusión, se observa que el grupo 1 es el que presenta temperaturas más estables que el resto en invierno y verano, mientras que los otros dos grupos registran amplitudes térmicas mayores.

\section{Demanda de energía para la climatización en función del comportamiento térmico de cada MU}

A partir del relevamiento efectuado - que incluye las temperaturas urbanas, los correspondientes GD oficiales y GD experimentales obtenidos para cada grupo (descriptos en la sección denominada Análisis de zonas de comportamiento térmico similar) y las características constructivas (ver Tabla 3) -, se calculan los resultados de la demanda de energía para climatizar los edificios correspondientes a los distintos MU estudiados (Tabla 7 y Figura 8).

Asimismo, en la Figura 9 se presentan las diferencias obtenidas entre la demanda de energía calculada a partir de los datos climáticos oficiales y los experimentales, tanto para invierno como para verano. 
Figura 7 - Temperaturas promedio de los tres grupos identificados (7 días) en invierno (izquierda) y verano (derecha) - se indica la temperatura media esperada en invierno $\left(18^{\circ} \mathrm{C}\right)$ y en verano $\left(23^{\circ} \mathrm{C}\right)$
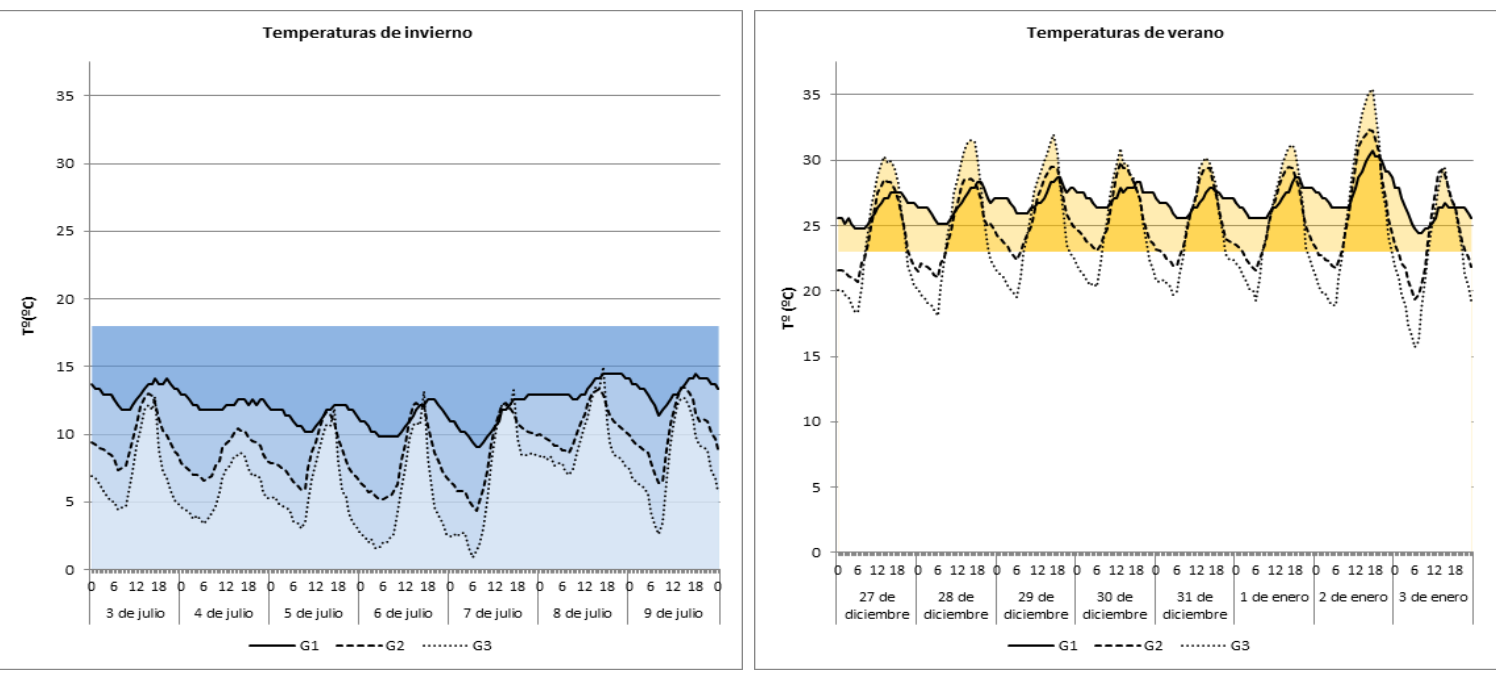

Tabla 7 - Cálculos de GD y Q a partir de GD oficiales (OALP) y 'experimentales'

\begin{tabular}{|c|c|c|c|c|c|c|c|c|c|c|c|}
\hline \multirow[b]{2}{*}{ MU } & \multicolumn{7}{|c|}{ DATOS DE CÁLCULO } & \multicolumn{2}{|c|}{ INVIERNO } & \multicolumn{2}{|c|}{ VERANO } \\
\hline & $\sum_{0}^{P}$ & 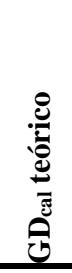 & 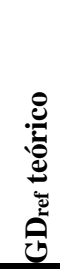 & 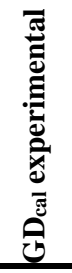 & 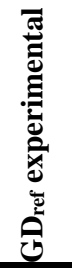 & 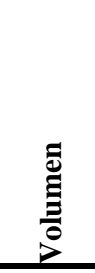 &  & 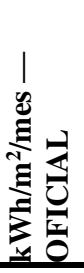 & 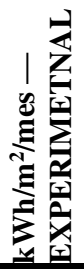 & نَ &  \\
\hline 01.Casco- La Loma & 2,0 & \multirow{10}{*}{270} & \multirow{10}{*}{103} & 131 & 146 & 25119 & 8373 & 38,9 & 18,9 & 14,8 & 21,0 \\
\hline 02.Casco-Circunvalación & 2,2 & & & \multirow{4}{*}{208} & \multirow{4}{*}{106} & 22542 & 7514 & 43,5 & 33,5 & 16,6 & 17,2 \\
\hline 03.Casco- Eje fundacional & 1,4 & & & & & 32775 & 10925 & 27,7 & 21,4 & 10,6 & 10,9 \\
\hline 04.City Bell-periferia & 1,9 & & & & & 8936 & 2103 & 51,4 & 39,6 & 19,6 & 20,3 \\
\hline 05.Gonnet-Periferia & 2,1 & & & & & 8825 & 2743 & 44,6 & 34,3 & 17,0 & 17,6 \\
\hline 06.Los Hornos- Periferia & 2,4 & & & \multirow{2}{*}{251} & \multirow{2}{*}{123} & 5292 & 1601 & 51,2 & 47,6 & 19,5 & 23,3 \\
\hline 07.San Carlos- Periferia & 2,0 & & & & & 16764 & 4332 & 49,6 & 46,1 & 18,9 & 22,5 \\
\hline 08.Tolosa- Periferia & 1,7 & & & 208 & 106 & 28599 & 6943 & 46,5 & 35,9 & 17,8 & 18,4 \\
\hline 09.Villa Elisa- Periferia & 2,2 & & & 251 & 123 & 13870 & 4250 & 45,9 & 42,7 & 17,5 & 20,9 \\
\hline 10.Villa Elvira- Periferia & 2,3 & & & 208 & 106 & 13999 & 4438 & 46,0 & 35,5 & 17,6 & 18,2 \\
\hline
\end{tabular}

Figura 8 - Demanda de energía mensual de invierno y de verano calculada con datos oficiales y experimentales

Demanda de energía de invierno

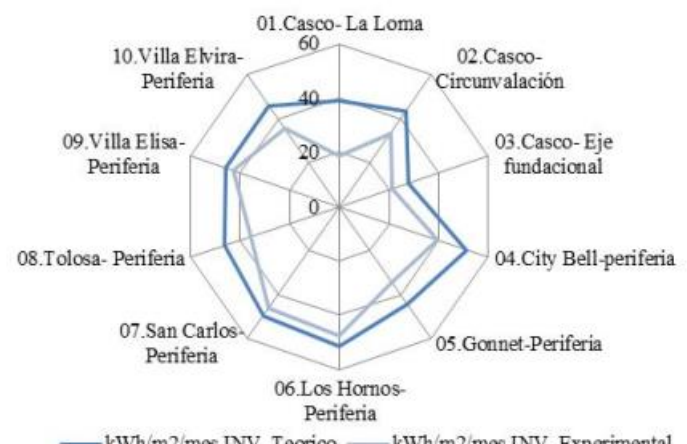

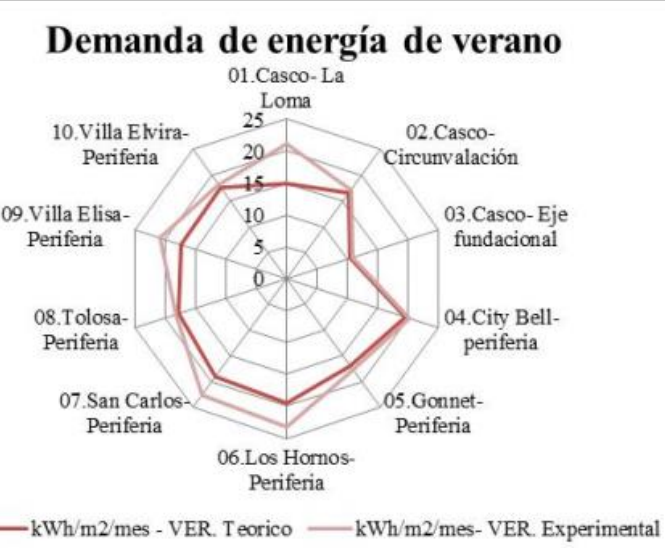


Figura 9 - Sobre y subestimación de la demanda de energía utilizando datos oficiales respecto de los experimentales

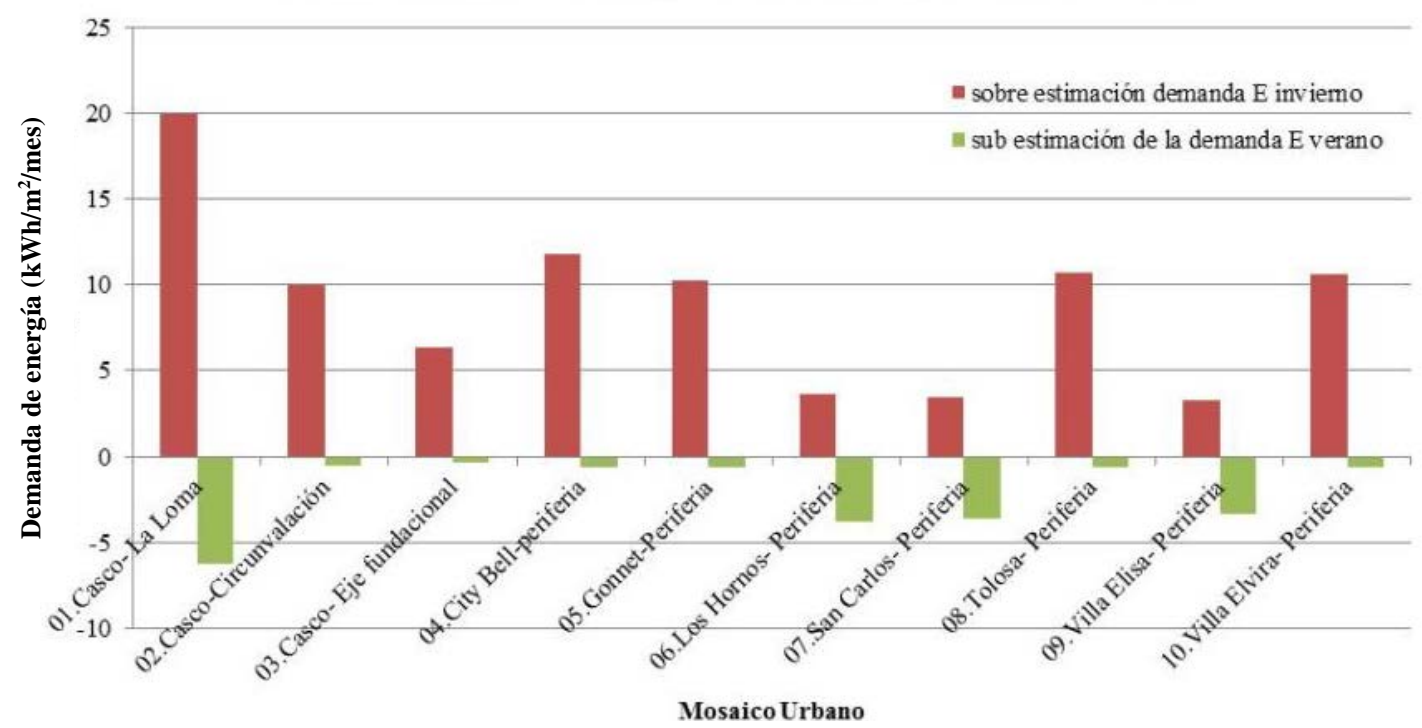

Si se analiza la demanda de energía de invierno (ver novena columna de la Tabla $7\left[\mathrm{kWh} / \mathrm{m}^{2} / \mathrm{mes}\right.$ INVIERNO OFICIAL]), se observa que las áreas críticas con consumos elevados son aquellas que tienen menor grado de compacidad o tejido urbano disperso, siendo estos los sectores 04 (City Bell), 06 (Los Hornos) y 07 (San Carlos). Existen áreas de compacidad media que presentan demandas de energía promedio, como los sectores 08 (Tolosa), 09 (Villa Elvira) y 10 (Villa Elisa); estas áreas tienen una superficie construida levemente mayor y presentan un tejido más compacto. Por su parte, los sectores de mayor densidad y compacidad (mosaicos del CF) son los que presentan menores demandas de energía por $\mathrm{m}^{2}$ de superficie construida.

Cuando se analiza la demanda de energía de invierno en función de los GD experimentales de cada mosaico (ver décima columna de la Tabla 7 [kWh $/ \mathrm{m}^{2} / \mathrm{mes}$ - INVIERNO EXPERIMENTAL]), se puede observar que ésta se reduce significativamente (ver Figura 8). Por su parte, en la Figura 8 se observan las sobrestimaciones de la demanda de energía para calefacción. En invierno, en las zonas de mayor consolidación y compacidad, la sobrestimación en función de los datos estadísticos oficiales es del 50\% (MU1). La mayoría de los sectores de la periferia urbana están sobreestimados en aproximadamente un 20\% (MU2, MU4, MU5, MU8 y MU10). Mientras que los mosaicos localizados muy cercanos al área rural y bañados presentaron resultados de demanda con GD experimentales cercanos a la demanda con GD oficiales (MU6, MU7 y MU9). Tengamos en cuenta que en Correa, De Rosa y Lesino (2008) también se obtuvieron sobreestimaciones máximas del orden del 50\%.

Por otro lado, podemos considerar que existen áreas urbanas en las que coinciden una mayor compacidad del tejido con una menor cantidad de $\mathrm{GD}_{\text {cal }}$, lo cual redunda en una menor demanda de energía. En consecuencia, presentan menor criticidad con respecto a la necesidad de reducir sus consumos, o lo que podríamos determinar cómo menor potencialidad de reducción energética específica. También existen áreas urbanas con mayor área de disipación vertical y horizontal, asociadas a una mayor cantidad de GD, que serían potencialmente acertadas para proponer medidas de mejoramiento térmico de su envolvente, ya que permitirían una importante reducción en su demanda de energía específica.

Al analizar la situación de verano, podemos concluir que las demandas de energía calculadas son más parejas. Éstas oscilan entre 10,9 y 23,3 $\mathrm{kWh} / \mathrm{m}^{2} / \mathrm{mes}$. Como ejemplo se puede observar que, en el cálculo teórico, el sector de mayor densidad tiene menor demanda energética que el resto de los sectores (01.Casco La Loma, con una demanda de 14,8 $\left.\mathrm{kWh} / \mathrm{m}^{2} / \mathrm{mes}\right)$. Sin embargo, la situación se invierte cuando consideramos los GD experimentales, ya que a partir de ellos aumenta ampliamente su demanda (21 $\mathrm{kWh} / \mathrm{m}^{2} / \mathrm{mes}$, subestimada en un 40\%). Sectores de la periferia (como 03.Los Hornos) tienen una demanda con datos oficiales de $19,5 \mathrm{kWh} / \mathrm{m}^{2} / \mathrm{mes}$, mientras que la experimental es de $23,3 \mathrm{kWh} / \mathrm{m}^{2} / \mathrm{mes}$ (subestimada en un 19\%). Otros sectores (como el 04.City Bell-periferia) tienen una demanda con datos oficiales de $19,6 \mathrm{kWh} / \mathrm{m}^{2} / \mathrm{mes}$ y una experimental de $20,3 \mathrm{kWh} / \mathrm{m}^{2} / \mathrm{mes}$ (subestimada en 
un 3\%). En la bibliografía se observó que el uso de datos microclimáticos simulados para el cálculo de demanda en refrigeración en ciudades costeras de Chile y de Ecuador (PALME et al., 2017b) arrojó valores de subestimación de entre un 15 y un 200\%, muy superiores a los obtenidos en este trabajo.

En el caso del verano, entonces, las áreas de mayor compacidad y las áreas localizadas en colindancia con sectores rurales o de bañados son las que tienen mayor consumo calculado con datos experimentales, mientras que las zonas que se ubican entre estos dos extremos son las de consumos medios. Por lo expuesto, los sectores de la periferia presentan una demanda específica mayor con menores superficies construidas, lo que generaría una importante reducción de energía al aplicar medidas de mejoramiento. En la Figura 8 se muestra la subestimación en la demanda de energía para refrigeración utilizando los valores teóricos en lugar de los experimentales.

Al comparar el análisis para las dos estaciones críticas, verano e invierno, podemos concluir que los sectores de la periferia urbana tienen una demanda de energía específica mayor ( $\mathrm{kWh} / \mathrm{m}^{2} / \mathrm{mes}$ ), por lo cual presentan una mayor potencialidad de reducción ésta. A partir de dicho análisis podríamos asociar estos resultados obtenidos a las posibles medidas de mejoramiento que puedan ser aplicadas. En este sentido, las estrategias de eficiencia energética y de aprovechamiento de la energía solar en áreas de la periferia urbana se presentan con gran potencial debido a que se registran amplias superficies disponibles y escasas obstrucciones solares. Por otro lado, el mejoramiento de la calidad térmica de la envolvente edilicia para hacer eficiente el uso de energía para climatización, requiere de una mayor inversión (55-75 U\$S $/ \mathrm{m}^{2}$ ) debido a la existencia de mayores superficies de disipación, si bien dicha acción redunda en mayores ahorros de energía netos.

\section{Conclusiones}

En función de los aspectos desarrollados, se puede concluir que el análisis térmico urbano permite ajustar la información existente - en general calculada a partir de mediciones climáticas oficiales - y perfeccionar el cálculo de la demanda de energía urbana a través del uso de modelos simplificados que consideren las variaciones de temperatura intraurbana. En consecuencia, se entiende que la temperatura urbana representa una variable significativa a tener en cuenta. Si bien en este caso responde a un estudio particular, dicha lógica puede incorporarse a otras ciudades, permitiendo obtener nuevos resultados hasta el momento no estimados.
Asimismo, los antecedentes internacionales demostraron la importancia de su consideración, aunque, en general, se orientaron a mejorar el confort térmico de los corredores urbanos y no a determinar la reducción potencial de la demanda de energía en la edificación.

\section{Sobre la nueva información térmica obtenida}

Tal como se analizó, se observan amplias diferencias térmicas en los distintos sectores urbanos de la ciudad de La Plata. En invierno las temperaturas intraurbanas medias oscilan entre 9,3 ${ }^{\circ} \mathrm{C}$ y $14,3^{\circ} \mathrm{C}$, siendo que las más bajas corresponden a la periferia norte y las más elevadas a la zona central del CF. Si bien no se observa una relación directa entre el FOS o el FOT y las temperaturas medias, se puede concluir que los sectores con menores temperaturas responden a la cercanía con áreas abiertas rurales poco forestadas o regiones de bañados. En el verano las temperaturas intraurbanas medias oscilan entre $24,3{ }^{\circ} \mathrm{C}$ y $27,6{ }^{\circ} \mathrm{C}$ demostrando menor amplitud térmica entre los sectores urbanos. En este sentido, para terminar de explicar estos comportamientos, se deberá ampliar el análisis a partir de consideraciones tales como el nivel de reparo de los vientos que provocan las masas arbóreas y la compacidad de los sectores urbanos, entre otros aspectos.

\section{Sobre la potencialidad de la reducción de la demanda de energía para climatización}

Como verificación de las hipótesis iniciales, existen diferencias sustanciales en la demanda de energía si se utilizan sólo las temperaturas medias oficiales de la ciudad. En invierno, en las zonas de mayor consolidación y compacidad, se calculó una sobrestimación de la demanda en función de los datos estadísticos oficiales del orden del 50\% y, en la mayoría de los sectores de la periferia urbana, del orden del $20 \%$.

En cuanto a la demanda de energía de los MU próximos a áreas rurales y bañados, calculados con los GD oficiales y GD experimentales, los resultados han sido similares en todos los casos. Con respecto al verano, se encontraron subestimaciones del orden del $40 \%$ en las áreas de mayor consolidación, mientras que, en las periferias cercanas a las áreas rurales, fue del 19\% y, en las zonas intermedias localizadas entre estos dos sectores, fue del 3\%, cercano a los valores oficiales.

En cuanto al estudio del consumo de energía específica ( $\left.\mathrm{kWh} / \mathrm{m}^{2} / \mathrm{mes}\right)$, se concluyó que existe un mayor potencial de reducción de la demanda de 
energía para climatización en los sectores periféricos respecto a las áreas centrales o de consolidaciones medias de la ciudad. Dichos sectores son aquellos que también permiten la incorporación de estrategias de sustitución energética basadas en el aprovechamiento de la energía solar, ya que cuentan con mayor disponibilidad del recurso debido a la dispersión edilicia y a su baja densidad. En tanto, en invierno los sectores centrales y más densos cuentan con beneficios en cuanto a la morfología urbana y, en consecuencia, a la condición climática exterior, minimizando la demanda de energía; ya en verano se requiere una atención especial, dado que se han registrado situaciones críticas de temperaturas elevadas.

\section{Referencias}

BREA, B. et al. Diagnóstico Energético del Sector Residencial de La Plata: interacción de variables energéticas y sociodemográficas. Estudios del Hábitat, v. 12, p. 7-23, 2014.

BROUSSE, O. et al. WUDAPT: an efficient land use producing data tool for mesoscale models? Integration of urban LCZ in WRF over Madrid. Urban Climate, v. 17, p. 116-134, 2016.

BUENO B. et al. The Urban Weather Generator. Journal of Building Performance Simulation, v. 6, n. 4, p. 269-281, 2013.

CAI, M. et al. Investigating the Relationship Between local Climate Zone and Land Surface Temperature Using an Improved WUDAPT Methodology: a case study of Yangtze River Delta, China. Urban Climate, in press, 2017.

CHEVEZ, P. et al. Application of the K-Means Clustering Method for the Detection and Analysis of Areas of Homogeneous Residential Electricity Consumption at the Great La Plata region, Buenos Aires, Argentina. Sustainable cities and Societies, v. 32, p. 115-129, jul. 2017.

CORREA, E.; DE ROSA, C.; LESINO, G. Urban Heat Island Effect on Heating and Cooling Degree Day's Distribution in Mendoza's Metropolitan Area and Environmental Costs. In: EUROSUN INTERNATIONAL CONFERENCE ON SOLAR HEATING, COOLING AND BUILDINGS, 13., Lisboa, 2008. Proceedings... Lisboa: Sociedade Portuguesa de Energia Solar, 2008.

DE RIDDER, K. et al. UrbClim: a fast urban boundary layer climate model. Urban Climate, v. 12, p. 21-48, jun. 2015.
DEGREE DAYS DIRECT LD. How Degree Days

Are Computed. Disponíble:

$<$ http://www.vesma.com/ddd/ddcalcs.htm].

Acceso: 15 nov. 2007.

\section{DÍSCOLI, C. Metodología Para el Diagnóstico}

Urbano-Energético-Ambiental en

Aglomeraciones Intermedias: el caso del gran La Plata. La Plata: Editorial Universitaria La Plata, 2009.

DÍSCOLI, C.; MARTINI, I. Desarrollo de Tecnologías y Pautas Para el Reciclado Masivo de la Envolvente Edilicia Residencial Orientado al Uso Racional y Eficiente de la Energía en Áreas Urbanas. 2012. Proyecto de Investigación Plurianual (PIP) 112-2011-0100097 - CONICET, La Plata.

FABBRI, K. et al. Outdoor Comfort: the ENVIBUG tool to evaluate PMV values point by point. Energy Procedia, v. 111, p. 510-519, 2017.

GALLINELLI, P.; CAMPONOVO, R; GUILLOT, V. CityFeel: micro climate monitoring for climate mitigation and urban design. Energy Procedia, v. 122, p. 391-396, 2017.

GASPARI, J.; FABBRI, K. A Study on the Use of Outdoor Microclimate Map to Adress Desgn Solutions For Urban Regeneration. Energy Procedia, v. 111, p. 500-509, 2017.

HURTADO, M. (Coord). Análisis Ambiental del Partido de La Plata: aportes al ordenamiento territorial. instituto de geomorfología y suelos. La Plata: Centro de Investigaciones de Suelos y Aguas de Uso Agropecuario, Municipalidad de La Plata, Consejo Federal de Inversiones, 2006.

INSTITUTO ARGENTINO DE RACIONALIZACIÓN DE MATERIALES.

IRAM 11603: acondicionamiento térmico de edificios: clasificación bioambiental de la República Argentina. Buenos Ayres, 2012.

INSTITUTO ARGENTINO DE RACIONALIZACIÓN DE MATERIALES. IRAM 11549: aislamiento térmico de edificios: volabulario. Buenos Ayres, 2002.

\section{INSTITUTO ARGENTINO DE} RACIONALIZACIÓN DE MATERIALES.

IRAM 11604: aislamiento térmico de edificios: verificación de sus condiciones higrotérmicas: ahorro de energía en calefacción: coeficiente volumétrico $\mathrm{G}$ de pérdidas de calor: cálculo y valores límite. Buenos Ayres, 2001. 
INSTITUTO ARGENTINO DE RACIONALIZACIÓN DE MATERIALES.

IRAM 11605: acondicionamiento térmico de edificios: condiciones de habitabilidad en edificios: valores máximos de transmitancia térmica en cerramientos opacos. Buenos Ayres, 1996a.

\section{INSTITUTO ARGENTINO DE \\ RACIONALIZACIÓN DE MATERIALES.}

IRAM 11659-1 Y 2: acondicionamiento térmico de edificios: verificación de sus condiciones higrotérmicas: ahorro de energía en refrigeración: parte 2: edificios para viviendas. Buenos Ayres, 1996b.

INSTITUTO DE ARQUITECTURA SOLAR. Plan Piloto de Evaluaciones Energéticas de la zona Capital Federal y Gran Buenos Aires. Investigación realizada por Concurso Nacional organizado por la CIC y Secretaría de Energía de la Nación (AUDIBAIRES). La Plata, 1987. Contrato SE-N1-1399/83 y SE N1 1399/83.

INSTITUTO NACIONAL DE ESTADÍSTICAS Y CENSOS. Proyecciones por Departamento. Cuadros Estadísticos: años 2010-2025. Buenos Ayres, 2017.

\section{INTERNATIONAL ENERGY AGENCY.} Indicadores de Eficiencia Energética: bases esenciales para el establecimiento de políticas. Paris, 2015. Disponible en:

$<$ https://www.iea.org/publications/freepublications /publication/EnergyEfficiencyVespagnol_epdf.pdf >. Acceso: 15 nov. 2017.

LEE, D.; OH, K. Classifying Urban Climate Zones (UCZs) Based on Statistical Analyses. Urban Climate, in press, 2017.

LI, J.; WANG, J.; WONG, N. Urban MicroClimate Research in High Density Cities: case study in Nanjing. In: INTERNATIONAL CONFERENCE ON COUNTER MEASURES TO URBAN HEAT ISLAND (UHI), 4., Singapore, 2016. Procedia... London: Elsevier, 2016.

MINISTERIO DE ENERGÍA Y MINERÍA. Balance Energético Nacional 2016. Buenos Ayres, 2017. Disponible en:

<http://www.energia.gob.ar/contenidos/verpagina. php?idpagina=3366>. Acceso: 15 abr. 2017.

OCHOA, J. M., SERRA, R. Microclimatic Analysis of Some Urban Scenarios, Environmental Friendly Cities. In: PLEA'98, Lisbon, 1998.

Proceedings... Lisbon, 1998.
PALME, M. et al. From Urban Climate to Energy Consumption: enhancing building performance simulation by including the urban heat island effect. Energy and Buildings, v. 145, p. 107-120, 2017b.

PALME, M. et al. Urban Weather Data and Building Models for the Inclusion of the Urban Heat Island Effect in Building Performance Simulation. Journal Data in Brief, v. 14, p. 671675, 2017a.

PALME, M.; CARRASCO C.; GALVEZ M. A. Estimación del Riesgo de Sobrecalentamiento y del Potencial de Refrigeración Por Ventilación Natural de Viviendas Unifamiliares en Ciudades Costeras de Chile. Revista Hábitat Sustentable, v. 6, n. 2, p. 52-61, 2016.

PARK, K. et al. Analysis of Micro-Climate on the Programs of Urban Infrastructure Regeneration in J City, Republic of Korea. Urban Forestry \& Urban Greening, v. 27, p. 43-49, 2017.

PÉREZ LÓPEZ, C. Técnicas de Análisis

Multivariante de Datos: aplicaciones con SPSS. Madrid: Pearson Educación, 2004.

RODRIGUEZ ALVAREZ, J. Urban Energy Index for Buildings (UEIB): a new method to evaluate the effect of urban form on buildings' energy demand. Landscape and Urban Planning, v. 148, p. 170-187, 2016.

ROSENFELD, E. Políticas de Uso Racional de la Energía en Áreas Metropolitanas y Sus Efectos en la Dimensión AMBIENTAL (URE-AM). La Plata, 1999. PID- Nº 4717/96 y PICT 98 N $13-$ 04116/99.

SOSA, M.; CORREA, E.; CANTÓN, M. ¿En Qué Medida la Forma Urbana Afecta al Comportamiento Térmico Exterior? Un estudio para reducir la isla de calor urbana en una ciudad árida. In: ENCUENTRO NACIONAL SOBRE CIUDAD, ARQUITECTURA Y CONSTRUCCIÓN SUSTENTABLE, 1., La Plata, 2016. Actas... La Plata: UNLP, 2016b.

SOSA, M.; CORREA, E.; CANTÓN, M. Morfología Urbana y Comportamiento Térmico de Canales viales: desarrollo de un modelo predictivo para temperaturas máximas. Revista Urbano, v. 33, p. 66-75, 2016a.

VERDONCK, M. et al. Influence of Neighbourhood Information on 'Local Climate Zone' Mapping in Heterogeneous Cities. International Journal of Applied Earth Observation and Geoinformation, v. 62, p. 102113, 2011. 
VIEGAS, G. Desarrollo Metodológico a Partir de Mosaicos Urbanos Para Evaluar la Eficiencia Energética y el Aprovechamiento de la Energía Solar en el Marco de la Sustentabilidad Urbana. Ambiente Construido, Porto Alegre, v. 11, n. 2, p. 139-155, abr./jun. 2011.

VIEGAS, G.; SAN JUAN, G. Energy and Environmental Aptitude (EEA) to Assess Solar Energy Exploitation in Cities. Resources and Environment, v. 2, n. 5, p. 185-192, 2012.

WUDAPT. The World Urban Database and Access Portal Tools project. 2017. Disponible: <http://www.wudapt.org/>. Acceso : 15 nov. 2017.
ZÖLCH, T. et al. Using Green Infrastructure for Urban Climate-Proofing: an evaluation of heat mitigation measures at the micro-scale. Urban Forestry \& Urban Greening, v. 20, p. 305-316, 2016.

\section{Agradecimientos}

Agradecemos la información proporcionada por el Departamento de Sismología e Información Meteorológica, de la FCAG, UNLP.

También agradecemos el premio Maurício Roriz de Melhor artigo científico, otorgado en el XIV Encontro Nacional de Conforto no Ambiente Construído, organizado por la Associação Nacional de Tecnologia do Ambiente Construído (ANTAC).

\section{Graciela Melisa Viegas}

Instituto de Investigaciones y Políticas del Ambiente Construido, Facultad de Arquitectura y Urbanismo | Universidad Nacional de La Plata | Calle 47, 162 | La Plata - Buenos Aires - Argentina | CP 1900 | Tel.: +54 (221) 4236589 | E-mail: gachiviegas@yahoo.com.ar

\section{Pedro J oaquín Chevez}

Instituto de Investigaciones y Políticas del Ambiente Construido, Facultad de Arquitectura y Urbanismo | Universidad Nacional de La Plata | E-mail: chevezpedro@gmail.com

\section{Gustavo Alberto San J uan}

Instituto de Investigaciones y Políticas del Ambiente Construido, Facultad de Arquitectura y Urbanismo | Universidad Nacional de La Plata | E-mail: gustavosanjuan60@hotmail.com

\section{Carlos Alberto Díscoli}

Instituto de Investigaciones y Políticas del Ambiente Construido, Facultad de Arquitectura y Urbanismo | Universidad Nacional de La Plata | E-mail: discoli@rocketmail.com

\section{Revista Ambiente Construído}

Associação Nacional de Tecnologia do Ambiente Construído

Av. Osvaldo Aranha, 99 - 3o andar, Centro

Porto Alegre - RS - Brasil

CEP 90035-190

Telefone: +55 (51) 3308-4084

Fax: +55 (51) 3308-4054

www. seer. ufrgs. br/ ambienteconstruido

E-mail: ambienteconstruido@ufrgs.br 